\title{
Vascular smooth muscle cells in atherosclerosis
}

Gemma L. Basatemur $^{1}$, Helle F. Jørgensen ${ }^{1}$, Murray C.H. Clarke ${ }^{1}$, Martin R. Bennett ${ }^{1}$, and Ziad Mallat ${ }^{1,2 *}$

${ }^{1}$ Division of Cardiovascular Medicine, Department of Medicine, University of Cambridge, Cambridge, UK. ${ }^{2}$ INSERM U970, Paris Cardiovascular Research Center, Paris, France;

Université Paris Descartes, Sorbonne Paris Cité, Paris, France.

*Address for correspondence: Ziad Mallat, British Heart Foundation Laboratory of Cardiovascular Medicine, University of Cambridge, West Forvie building, Robinson Way, Cambridge, CB2 0SZ, UK. zm255@medschl.cam.ac.uk 


\begin{abstract}
Vascular smooth muscle cells (VSMCs) are a major cell type present at all stages in atherosclerotic plaques. According to the 'response to injury' and 'vulnerable plaque' hypotheses, contractile VSMCs recruited from the media undergo phenotypic conversion to proliferative synthetic cells that elaborate extracellular matrix to form the fibrous cap and hence stabilise plaques. However, recent lineage tracing studies have highlighted flaws in the interpretation of former studies, revealing these to have underestimated both the content and functions of VSMCs in plaques, and have thus challenged our view on the role of VSMCs in atherosclerosis. It is now evident that VSMCs are even more plastic than previously recognised, and can adopt alternative phenotypes including cells resembling foam cells, macrophages, mesenchymal stem cells, and osteochondrogenic cells, which could contribute both positively and negatively to disease progression. In this review, we present the evidence for VSMC plasticity and summarise the roles of VSMCs and VSMC-derived cells in atherosclerotic plaque development and progression. Correct attribution and spatio-temporal resolution of clinically beneficial and detrimental processes will underpin the success of any therapeutic intervention aimed at VSMCs and their derivatives.
\end{abstract}

\title{
Introduction
}

Atherosclerosis is the formation of plaques containing lipid, cells, debris and scar tissue in the intima of arteries. As the main pathological process underlying myocardial infarction, angina, heart failure and stroke, atherosclerosis has been the leading cause of morbidity and mortality in the Western world for over half a century and is now the top cause of death globally ${ }^{1}$. A significant role for vascular smooth muscle cells (VSMCs) in atherosclerosis was established in the 1960s - as soon as electron microscopy made it possible to identify smooth muscle-like cells in the media of normal arteries ${ }^{2}$, and it was ascertained that the majority of cells in atherosclerotic plaques had characteristics of VSMCs but with altered phenotypes $^{3-5}$. However, the perception of how VSMCs contribute to plaque development, remodelling and stabilisation has changed substantially over the last half-century (Box 1), and recent studies have questioned long-standing assumptions about the identity of cells in plaques, demanding a re-evaluation of the role of VSMCs in atherosclerosis.

\section{Identification of VSMCs}

VSMCs are defined based upon anatomical localisation (i.e. within the vasculature) and functionality; in healthy arteries VSMCs are located in the medial layer where they are responsible for arterial contraction and production of extracellular matrix (ECM), and play important roles in compliance and elastic recoil in response to changing haemodynamic conditions. VSMC functions are key determinants of the properties of vessels throughout the arterial tree; VSMC-derived elastin is crucial for elastic recoil in large elastic arteries (such as the aorta), whilst VSMC contraction is largely responsible for modulating arterial diameter in muscular arteries and arterioles (the latter being of great importance to systemic arterial resistance). Functionality is usually inferred from a combination of characteristics, including morphology and expression of 'VSMC-specific' function-associated markers (which are typically proteins and glycosaminoglycans). In healthy arteries, VSMCs are fusiform-shaped cells that express contractile proteins (including smooth muscle alpha actin ( $\alpha \mathrm{SMA}$ ) and smooth muscle myosin heavy chain (SMMHC) which are organised into myofilaments) and 
secrete ECM macromolecules (including elastins, collagens and proteoglycans). Most studies to date have relied on these markers ${ }^{6-9}$ or gene expression profiles ${ }^{10}$ for identification of VSMCs. However, as a necessary corollary of their role in tissue homeostasis and repair, VSMCs exhibit considerable phenotypic plasticity in atherosclerosis, in response to injury, and upon culture in vitro, which is often accompanied by marked changes in cell morphology and expression of 'VSMC-specific' markers. Hence, definition of cell-type based on functionality or 'specific' markers as a proxy for cell identification is problematic, and has confounded studies on the true extent of the role of VSMCs in atherosclerosis ${ }^{11}$.

Developments in genetic engineering have enabled specific labelling of VSMCs in mice, making fate mapping and lineage tracing of VSMCs possible. For example, inducible VSMC labelling systems (such as a tamoxifen inducible-recombinase driven by 'VSMC-specific' gene promoters (typically MYH11 ${ }^{12}$ or $\left.T A G L N^{13-15}\right)^{16}$ combined with reporter proteins ${ }^{17,18}$ ), result in specific and stable labelling of VSMCs at baseline and enable unambiguous tracing of VSMCs and VSMC-derived cells during atherogenesis, even when VSMC characteristics may otherwise be lost or gained ${ }^{11,17-24}$. This elegant approach has led to important advances in our understanding of the functional consequences of developmental origin, plasticity, clonality and ultimately the fate of VSMCs in plaques, providing evidence for a more complex and prominent role for VSMCs and VSMC-derived cells in atherosclerosis.

\section{Origin of VSMCs}

VSMCs are derived from multiple distinct progenitors in embryogenesis (detailed in Box 2), with little or no mixing between different lineages ${ }^{25-27}$, resulting in anatomical segmentation across the arterial tree. Furthermore, there is evidence for positional identity among VSMCs along the anterior-posterior, dorso-ventral, and right-left axes of the embryo ${ }^{28-30}$. Embryonic lineage can have important functional consequences; for example, VSMCs show lineagedependent responses to important signalling pathways such as TGF- $\beta^{31,32}$, PDGF $^{33}$, MRTFB $^{34,35}$, NF- $\mathrm{KB}^{36}$ and angiotensin $\mathrm{II}^{37}$. These findings exemplify a fundamental limitation in defining VSMCs on the basis of 'VSMC-specific' function-associated markers, which may be similarly expressed in all VSMC lineages (potentially evoked through different pathways that converge on the same set of 'VSMC-specific' genes, as detailed in Box 3), whilst different VSMC lineages may have distinct functional characteristics.

Lineage tracing studies have unambiguously demonstrated that VSMCs contribute substantially to plaque formation in murine models of atherosclerosis, generating $30-70 \%$ of all plaque cells ${ }^{11,18-20,22,23}$. In particular, most $\alpha$ SMA positive cells within the fibrous cap are VSMC lineage label positive, refuting earlier ideas ${ }^{38,39}$ that bone marrow-derived cells generate $\alpha$ SMA positive cells ${ }^{40-42}$. VSMC-derived cells that express mesenchymal stem cell markers (in particular Sca1) have also been identified in the healthy media ${ }^{43}$ and in plaques $^{11,43}$, and may represent a plastic intermediate population that is readily responsive to inflammation and capable of generating contractile or phenotypically switched VSMCs ${ }^{43}$. However, these studies do not rule out a contribution from other sources of progenitors to plaque VSMCs (Box 2).

Evidence for clonality (discussed below) of VSMCs and VSMC-derived cells in plaques indicates that the majority of plaque cells derive from recruitment and proliferation of local VSMCs, while the anatomical distribution of different developmental origins of VSMCs (and perhaps other cell types, such as pericytes and endothelial cells) may contribute to the 
anatomical distribution of atherosclerosis susceptibility ${ }^{44}$. This idea is supported by the finding that segments of aorta from atherosclerosis-prone and -resistant regions maintain their atherosclerosis susceptibility upon transplantation to alternative sites ${ }^{45}$. Definitive evidence of similar anatomical segmentation of VSMCs populations in humans is currently lacking, but supported in part by studies showing that human arteries are composed of clonal patches of VSMCs ${ }^{46-48}$. Furthermore, advances in understanding development of different VSMC lineages in vivo have led to generation of VSMCs from stem cells ${ }^{49}$, which will facilitate better disease modelling in human cells in vitro ${ }^{50}$.

\section{Plasticity of VSMCs}

VSMCs display a fully functional, differentiated phenotype in healthy vessels, yet retain remarkable plasticity. De-differentiation, modulation, or phenotypic switching of VSMCs is characterised by reduced myofilament density and lower expression of contractile proteins. De-differentiated VSMCs upregulate expression of ECM components and ECM-remodelling enzymes, have increased levels of secretory organelles, and express pro-inflammatory cytokines $^{51}$. Consequently, phenotypically-switched VSMCs are often referred to as 'synthetic', whilst VSMCs expressing high levels of contractile proteins are generally described as 'contractile' (although these definitions imply explicit functional changes that are usually only inferred and very rarely quantified). Activation of VSMC proliferation and migration has also been associated with the synthetic, de-differentiated state, but coordinated regulation of these processes has not been documented and mitotic VSMCs with high levels of contractile proteins have been observed ${ }^{52,53}$.

Phenotypic switching is a reversible process, at least in the early stages. For example, a general, transient loss of contractile protein expression is observed after vascular injury, followed by reestablishment of the contractile phenotype after vessel repair ${ }^{54}$. VSMCs displaying phenotypes ranging from contractile to synthetic states have also been observed both in vivo ${ }^{53}$ and in VSMC cultures in vitro ${ }^{55,58}$, illustrating that phenotypic switching is not a binary process. VSMC heterogeneity in morphology and gene expression ${ }^{43,56}$ is also seen in healthy vessels, including detection of rare atypical VSMCs marked by Sca1/Ly6a, that express phenotypic switch-associated genes ${ }^{43}$. At the molecular level, VSMC phenotype is governed by regulatory transcription factors (including myocardin/SRF ${ }^{57}$ and $\mathrm{KLF}^{11}$ ), which integrate input from the environment (including growth factors, cytokines, lipid mediators, contact with the ECM and other cells) and is regulated at multiple levels, including epigenetic mechanisms (summarised in Box 3).

Lineage tracing studies have revealed that VSMCs exhibit greater than anticipated plasticity in atherosclerosis (Table 1). Within plaques a large proportion of reporter-expressing VSMC-derived cells do not have detectable levels of the contractile smooth muscle cell marker $\alpha \mathrm{SMA}^{11,20}$. Instead, some plaque reporter-expressing cells were positive for Mac- $3^{20}$, Lgals $3^{11}$ and $C D 68{ }^{17}$ - markers that have been previously used to study macrophages in atherosclerosis. Stimulation of VSMCs in vitro with cholesterol similarly induces expression of macrophage-associated genes ${ }^{58,59}$ and promotes a phagocytic phenotype ${ }^{11}$. Human VSMC-derived plaque cells were also found to express $\mathrm{CD} 8^{11}$, consistent with previous studies co-detecting CD68 and $\alpha$ SMA in human plaque cells ${ }^{60,61}$. These results support the hypothesis proposed by Wissler in $1968^{62}$ that at least a subset of foam cells are VSMCderived. This should be considered when interpreting studies on macrophage function, which rely only on marker expression. Similarly, VSMCs have been proposed to generate 
osteochondrogenic and mesenchymal stem cell-like plaque cells based on expression of mineralising ECM proteins ${ }^{63,64}$ and Sca1/Eng ${ }^{11}$ respectively. Expanded plasticity of VSMCs in atherosclerosis was confirmed by transcriptional profiling of individual VSMC-lineage plaque cells, revealing subpopulations of cells expressing Ly6a/Sca1, CD68 and Sox9/Chad ${ }^{43}$.

\section{Clonality of VSMCs}

The combination of multi-colour recombination markers (such as the confetti or rainbow system $^{18,22}$ ) with genetic lineage tracing of VSMCs has demonstrated that, surprisingly, mouse VSMC-derived plaque cells are generated by clonal expansion of relatively few cells within the vessel wall $17,20,22,23$. In contrast, most medial cells do not contribute to mouse plaque formation and the role of VSMC migration independent of proliferation is limited ${ }^{20}$. Indeed, phenotypically distinct VSMC-derived plaque cells are generated from a common 'ancestor'. Observations of plaques at different timepoints suggest that, in mice, VSMCs first generate the cap followed by adoption of switched phenotypes in the lesion core ${ }^{23}$, but this remains to be experimentally tested.

The molecular mechanisms underlying clonality are yet to be established, but macrophage secreted factors have been implicated. For example, bone-marrow transplantation from integrin $\beta 3$-deficient mice into ApoE null mice results in polyclonal plaque VSMCs and VSMC-derived cells ${ }^{23}$, whilst conditioned media from integrin $\beta 3$-deficient macrophages is more mitogenic to VSMCs than conditioned media from wild-type macrophages ${ }^{23}$. Early stage cap VSMCs are highly proliferative and express $\alpha$ SMA, SMMHC, and importantly PDGFR $\beta^{23}$, akin to the primed PDGFR $\beta$-positive VSMC progenitors reported in models of hypoxia-induced pulmonary hypertension, which clonally expand in a PDGF-dependent manner ${ }^{65,66}$. This highlights a potential role for PDGF signalling in clonal expansion of VSMCs, and demonstrates that the study of VSMC clonal expansion in other vascular conditions ${ }^{20,65,67}$ may be relevant for further mechanistic dissection in atherosclerosis.

The small number of VSMCs contributing to lesion formation raises the question of whether disease-associated proliferation results from activation of specific cells that are primed to respond to injury (discussed in ref ${ }^{68}$ ). Supporting this idea, transcriptional profiling of VSMCs from healthy blood vessels revealed significant heterogeneity in expression of genes associated with vascular disease, suggesting the existence of VSMC subtypes ${ }^{43,56}$.

Alternatively, clonality may rely on selection of VSMCs with equal plasticity, based on location (e.g. proximal to breaks in the internal elastic lamina and/or mitogenic signals) or differential capacity for survival or senescence (see below). It has also been speculated that pathways of lateral inhibition may be operating, as is common in development ${ }^{22}$. Importantly, these possibilities are not mutually exclusive, and the underlying mechanism is likely genetic (somatic mutations) and/or epigenetic changes in the expanded VSMCs relative to non-expanded VSMCs.

It is well documented that somatic mutations underlie clonal expansion both in malignancy and in non-malignant tissues as a consequence of aging ${ }^{69}$. Indeed, the acquisition of a particular set of somatic mutations, linked to clonal expansion, in myeloid progenitor cells has recently been shown to be associated with increased risk of atherosclerosis ${ }^{70}$. Therefore, it is reasonable to suggest that similar mechanisms may underlie clonal expansion of VSMCs in atherosclerosis. Indeed, when clonal expansion of VSMCs was first described in plaques it 
was likened to a smooth muscle cell tumour ${ }^{46}$. Epigenetic changes may influence clonal expansion of VSMCs secondary or independently of somatic mutations. Such changes may reflect differences in VSMC lineage, environmental stimuli, or stochastic events.

Whilst lineage tracing has provided the most robust evidence yet for clonality of VSMCs in plaques, the concept that most plaque VMSCs derive from clonal expansion, attributed to Benditt and Benditt ${ }^{46}$, has long been discussed ${ }^{47}$, particularly in the context of replicative senescence $^{71}$.

\section{VSMC Senescence}

Senescence is a protective mechanism that induces cell cycle arrest to prevent transmission of defects to progeny cells, particularly to stop malignant transformation ${ }^{72-74}$. Replicative senescence occurs after repeated cell division, typically after telomere erosion or damage, while induced senescence arises after oncogene activation, mitochondrial deterioration, DNA damage, or oxidative stress. A persistent DNA damage response (DDR) is the most unified pathway leading to senescence, with sensing by the Ataxia Telangiectasia Mutated (ATM) protein leading to 553 phosphorylation and upregulation of cell cycle inhibitors ${ }^{72-74}$. The cyclin-dependent kinase inhibitor (cdki) p21 drives initial cell cycle arrest, allowing repair of moderate DNA damage and re-entry into the cell cycle. However, prolonged arrest upregulates the cdki $\mathrm{p} 16^{\text {Ink4a }}$, leading to dephosphorylation of retinoblastoma protein $\mathrm{pRB}$, causing permanent cell cycle arrest ${ }^{72-74}$.

With every somatic cell division approximately 20bp or more is lost from the telomere ends of chromosomes. Thus, repeated cell division leads to critical shortening, telomeric erosion and loss of the protective Shelterin complex, which results in a persistent DDR that instigates senescence. VSMC senescence in vivo is likely driven by multiple pathways including DNA damage, mitochondrial deterioration, and oxidative stress - all present during atherosclerosis. Loss of autophagy can also drive VSMC senescence ${ }^{75}$. Replicative senescence is highly relevant in the context of plaque VSMC clonality, as to generate all the VSMC-derived cells in advanced plaques by clonal expansion would likely cause replicative senescence. In keeping with this, the telomeres of VSMCs in human plaques are markedly shortened, which correlates with disease severity ${ }^{76}$.

Most senescent cells develop altered secretory activities known as a senescence-associated secretory phenotype (SASP) ${ }^{77,78}$. Cells with SASPs release proinflammatory cytokines (such as IL-6, IL-1) and chemokines (such as IL-8, CCL2, CXCL1), growth factors (such as GCSF, bFGF), and proteases (including MMPs, PAI-1), conferring diverse activities ${ }^{78}$. IL-1 $\alpha$ is the key driver of the SASP ${ }^{79,80}$, with upstream expression controlled in part by ATM/ATRmediated liberation of GATA4 from p62-directed autophagy ${ }^{81}$ and/or an mTORC1-dependent pathway $^{82}$. In a physiological setting SASPs act as a molecular beacon that recruits and instructs immune cells to remove senescent cells (senescent surveillance ${ }^{83}$ ) before further mutation enables senescence bypass and, for example, re-initiation of tumour formation. However, uncleared senescent cells accumulate during aging and disease (perhaps due to a dysfunctional immune system or a suppressive milieu), and these generate chronic inflammation that could worsen outcome and/or drive atherosclerosis ${ }^{84}$.

Although VSMC senescence occurs in human plaques, proving the effects of senescent VSMCs is difficult, and hampered by technical difficulties in mouse models. For example, 
telomeres are approximately 10 times longer in mice than in humans, studying mouse SASPs in vitro is problematic ${ }^{85}$, and detecting senescence with the classic markers p16 and senescence associated $\beta$-galactosidase $(\mathrm{SA} \beta \mathrm{G})$ is also notoriously difficult in mice, particularly when both markers are expressed by macrophages in atherosclerotic plaques. Two main experimental approaches have been used to study the effect of VSMC senescence in atherosclerosis; modulation of senescence induction via the DDR, and clearance of naturally occurring senescent cells with 'senolytics'. For example, VSMC-specific expression of loss-of-function mutant TRF2 (a Shelterin subunit) led to increased DNA damage and VSMC senescence, with bigger plaques and necrotic cores, while gain-offunction TRF2 produced opposite effects ${ }^{86}$. Similarly, VSMCs that lack base excision repair activity have increased oxidative DNA damage and cell senescence, and promote increased plaque size ${ }^{87}$. In contrast, an intriguing recent study utilised electron microscopy to identify crystals proposed to be the product of $\mathrm{X}-\mathrm{Gal}$ cleavage by $\mathrm{SA} \beta \mathrm{G}^{84}$. This study reported more than $50 \%$ of all plaque cells to be senescent, including VSMCs, macrophages and endothelial cells ${ }^{84}$. Senescent cells appeared within 9 days of fat feeding, and both genetic and pharmacological elimination of $\mathrm{p} 16$ positive cells reduced plaque formation and progression $^{84}$. Although it is unclear which cells were senescent and removed by these treatments, this approach may open a new paradigm for atherosclerosis treatment.

\section{VSMCs in different stages of atherosclerosis}

Studies of plaque histology from human autopsy tissues have culminated in a scheme for classification of plaques that encapsulates the progression of atherosclerosis ${ }^{88,89}$ and, based on careful observations of plaque composition from human autopsy and animal models, it is clear that VSMCs are major contributors to plaque development at all stages (summarised in FIG. 1). However, their role and effects of VSMC proliferation or loss may differ according to the stage of atherogenesis.

\section{Pre-atherosclerosis}

Diffuse intimal thickenings (DITs), and intimal xanthomas (i.e. fatty streaks) are considered pre-atherosclerotic plaques, because they are common from birth ${ }^{90,91}$ and likely represent physiological adaptation to blood flow ${ }^{92}$. However, the relationship between intimal xanthomas and atherosclerosis is controversial because, although they localise to atherosclerosis-prone regions and some intimal xanthomas develop into atherosclerotic plaques, they are also found elsewhere and sometimes regress ${ }^{93-95}$. In contrast, DIT distribution in the young is similar to that of atherosclerotic plaques in later life ${ }^{90,96}$ and DITs are widely considered the most likely precursor to atherosclerotic plaques ${ }^{88}$.

Human DITs comprise VSMCs, proteoglycans and elastin, and lack macrophages and thrombus ${ }^{88,91,92}$. VSMCs in DITs exhibit clonality ${ }^{47,91}$, and are thought to originate from local medial VSMCs ${ }^{56}$. However, the latter is difficult to prove as many techniques for lineage tracing (e.g. reporter gene expression from a lineage-specific promoter), are limited to animal models, and most mammals (including mice) do not develop DITs ${ }^{97}$. VSMCs in DITs are heterogeneous, but most exhibit increased synthetic organelles (rough endoplasmic reticulum, ribosomes and mitochondria) compared to medial VSMCs ${ }^{98}$, consistent with switching to a synthetic phenotype, which is supported by decreased expression of contractile genes $^{99}$ and increased expression of ECM components ${ }^{100}$. VSMCs are thought to be the 
major source of the ECM in DITs, which accounts for much of the increase in thickness of the intima but, importantly for progression to atherosclerosis, DITs are rich in proteoglycans that are crucial for retention of apolipoproteins ${ }^{101}$. Furthermore, synthetic phenotype VSMCs metabolise lipid differently to contractile VSMCs, in part through decreased expression of cholesterol esterase and reduced cholesterol efflux transporter ABCA $1^{60,102}$, resulting in increased tendency towards foam cell formation ${ }^{103}$.

\section{Early atherosclerosis}

The first stage in atherosclerosis is the formation of pathological intima thickenings (PITs); the earliest recognised atherosclerotic plaque, which is characterised by the formation of an extra-cellular lipid pools deep in the intima, underlying abundant VSMCs and ECM ${ }^{88,89}$. DITs can, but do not always, progress to PITs (FIG. 2) ${ }^{104}$. Progression is promoted through a complex interplay between retention and oxidation of lipid, induction of inflammation, and VSMC proliferation, phenotype switching, and death.

The lipid pools(which is distinct from the necrotic pool of more advanced plaques) comprises lipids (including free cholesterol) amidst a proteoglycan (notably biglycan, versican and perlecan) and glycosaminoglycan (GAG, including hyaluronan) -rich ECM. As the predominant cell-type present in DITs, intimal VSMCs are regarded as the most important source of the ECM, and this is supported by analysis of the secretome of VSMCs in vitro ${ }^{105-}$ 109. The ECM has a central role in initiation of atherosclerosis, primarily through the interaction between the negatively charged side chains of proteoglycans (particularly chondroitin sulphate of biglycan and versican and heparin sulphate of perlecan ${ }^{110}$ ) with positively charged apolipoproteins (especially apolipoprotein B), which leads to the retention of plasma-derived lipoproteins ${ }^{101,111}$ - as described in the 'response to retention hypothesis'112,113. Transgenic mice over-expressing biglycan in VSMCs show more lipid retention and increased atherosclerosis than wild-type litter-mates ${ }^{114}$. Once retained in the intima, lipoproteins undergo modifications, including oxidation to OxLDL, which precedes the recruitment of macrophages ${ }^{115}$ and initiates the inflammatory response characteristic of atherosclerosis ${ }^{112}$. Further evidence for this series of events was provided by a recent study comparing DITs to PITs, in which extra-cellular lipid was found deep in the plaque, colocalising with $\alpha \mathrm{SMA}$-positive cells, ApoB, biglycan and versican, but not the more superficial (closer to the lumen) CD68 positive cells (likely macrophages) ${ }^{116}$.

Progression to PITs is accompanied by loss of $\alpha \mathrm{SMA}$, which is likely due to a combination of phenotypic switching of VSMCs ${ }^{11,18,23}$ and loss of VSMCs through cell death ${ }^{117,118}$. For example, uptake of OxLDL and formation of VSMC-derived foam cells has been linked to induction of VSMC death by apoptosis ${ }^{118}$, and free cholesterol in the lipid pool may be derived from dead VSMC ${ }^{119}$. The micro-calcification (speckles of $0.5-15 \mu \mathrm{m}$ ) sometimes observed within the lipid pool of PITs, typically close to the border with the media, may also be a consequence of VSMC apoptosis ${ }^{51}$.

Macrophages may be absent from early PITs ${ }^{89}$, but are a defining characteristic of late stage PITs and crucial to the progression of PITs to fibroatheromas. Lineage tracing studies have shown the macrophage marker-positive cells of early lesions in mice (which resemble intimal xanthomas) are mostly derived from recruited circulating monocytes ${ }^{23,120}$, and may also involve local resident macrophages ${ }^{121,122}$. However, co-expression of $\alpha$ SMA and CD68 in human plaques indicate that VSMCs also likely contribute significantly to the macrophage 
marker-positive cells in early plaques ${ }^{5,61}$. Monocytes are recruited to PITs through the expression of adhesion molecules (including selectins, ICAM1, VCAM1, CD31 ${ }^{123}$ ) and chemo-attractants, including chemokines (such as CCL5, CXCL1 and CCL2 ${ }^{120,124}$, which are secreted by VSMCs and ECs stimulated with inflammatory cytokines or OxLDL, in vitro ${ }^{125}$ ) and modified lipids (such as OxLDL ${ }^{126}$ ). Studies in animal models have collectively revealed an essential requirement for macrophages in the progression of atherosclerosis ${ }^{120,122,127,128}$, which is likely to involve effects on VSMC migration, proliferation (through production of factors such as $\mathrm{PDGF}^{129}$ ) and phenotype switching ${ }^{130}$.

\section{Late atherosclerosis}

PITs can progress to fibroatheromas (FIG. 3), characterised by the presence of a fibrous cap and a necrotic core, the origins of which are the extra-cellular lipid pool and insufficient efferocytosis (of dead VSMCs and macrophages) ${ }^{131-133}$. This phase of atherosclerosis (late PIT/early fibroatheroma) is dependent on extensive accumulation of macrophages on the luminal side of the lipid pool, where they phagocytose deposited lipids to become foam cells. In the absence of resolution, the ensuing inflammatory reaction is self-perpetuating; macrophages and VSMCs become foam cells, which die (mostly by apoptosis but potentially through other mechanisms, Box 4). Since the plaque milieu suppresses efferocytosis ${ }^{133-136}$, uncleared apoptotic cells subsequently undergo secondary necrosis with release of further inflammatory material, such as damage-associated molecular patterns (DAMPs) ${ }^{137}$. The accompanying healing response involves the formation of the fibrous cap, which, at least in the early stages, is a highly cellular region, rich in VSMC-derived $\alpha$ SMA-positive cells ${ }^{22,40-}$ 42 , amongst an altered ECM -that has decreased proteoglycan expression and an increase in the proportion of collagens (mostly type I and III).

In mice, the fibrous cap VSMCs are derived from medial VSMCs ${ }^{22,138}$ that have undergone migration and proliferation in response to cytokines and growth factors, such as PDGF, derived from macrophages and activated ECs ${ }^{23,129,139}$. This initial stage of VSMC recruitment is, at least in part, Oct4 dependent ${ }^{21}$. In humans, both pre-existing intimal and medial VSMCs may contribute to plaque VSMCs ${ }^{48}$. Definitive proof that VSMCs are responsible for the production of the fibrous cap ECM is lacking. However, this hypothesis is consistent with co-localization of collagen synthesis to VSMCs in the fibrous cap ${ }^{140}$, correlation of fibrous cap thickness with VSMC phenotype in mice ${ }^{11,21,141}$, and the correlation of fibrous cap stability with VSMC cell number in humans ${ }^{142}$. In addition, a recent study of VSMC-specific deletion of Col15a resulted in a greater than 70\% reduction in Col15a, supporting VSMCs as the major source of this collagen ${ }^{143}$. Further evidence that VSMCs are the major source of collagens comes from studies in vitro, including proteomic analysis of the secretome of lipid-loaded VSMCs ${ }^{109}$ and induction of collagen synthesis by VSMCs in culture by TGF- $\beta$, PDGF, IL-1, AngII, cholesterol, homocysteine and mechanical stretch $^{144,145}$.

VSMCs in the later stages of atherosclerosis have previously been thought to be entirely beneficial, for example by stabilising the plaque through elaborating the fibrous cap. However, lipid loading of VSMCs and altered interactions with the ECM lead to altered VSMC phenotype, and increased macrophage markers ${ }^{59}$. Indeed, VSMCs contribute between $30-70 \%$ of the macrophage marker-positive cells ${ }^{11,20}$ and similarly to foam cells ${ }^{146}$ in mouse plaques, and around 30-40\% of CD68 positive cells and 50\% of foam cells in humans ${ }^{11,60}$. VSMC-specific deletion of the transcription factor KLF4 reduces VSMC switching to 
macrophage marker-positive cells, and results in a marked increase in the thickness and $\alpha$ SMA-positive cell content of the fibrous cap ${ }^{11}$. Although these studies have shown that VSMCs can express macrophage markers, in vitro studies of the transcriptomes of VSMCs and macrophage-derived foam cells indicate they are functionally distinct, and that VSMCderived foam cells exhibit reduced phagocytic and efferocytic responses ${ }^{59}$. VSMCs have long been known to contribute to the inflammatory milieu of the plaque through recruitment of macrophages; however, these studies strongly suggest that VSMC-derived macrophagelike cells also directly affect plaque progression.

In early fibro-atheromas, calcification is observed as large granules in the necrotic core and surrounding ECM, resulting from a number of interrelated processes, including macrophage and VSMC-derived calcifying micro-vesicles ${ }^{147-149}$, release of apoptotic bodies ${ }^{150}$ or the activity of osteochondrogenic cells ${ }^{151}$. As the fibro-atheroma develops, micro-calcifications can coalesce into larger speckles and fragments that can form sheets or plates ${ }^{149}$ visible by tomography. Fragmentation of these sheets and fibrin encapsulation can lead to the formation of calcium nodules, which protrude into the vessel lumen and precipitate thrombosis $^{88}$. The extent of plaque calcification varies according to the vascular bed, and a recent study linked this to the different propensities of the local, developmentally distinct, VSMCs to undergo calcification ${ }^{152,153}$. VSMCs have long been linked to calcification ${ }^{150,154}$ and osteochondrogenic conversion in vitro is enhanced by plaque-like environmental cues, including phenotypic conversion ${ }^{155}$, apoptotic bodies ${ }^{150}$, oxLDL ${ }^{156}$, and inflammatory cytokines such as TNF $\alpha^{157}$, IL- $1^{158}$ and IL-18 ${ }^{159}$. Furthermore, specific genetic modulation of VSMC osteochondrogenesis in vivo leads to altered calcification in models of atherosclerosis $^{160-162}$. Most convincingly, however, recent studies have established that most of the osteochondreogenic precursors (Runx2/Cbfa1+ cells) and chondrocyte-like (type II collagen + ) cells of murine plaques are again VSMC-derived ${ }^{138}$.

\section{Clinical sequalae}

The major clinical sequelae of atherosclerosis are dependent on the anatomical site of the vascular bed involved (angina and myocardial infarction in coronary arteries; stroke in carotid arteries) and typically manifest as a result of thrombosis. The primary cause (accounting for around $60 \%$ to $70 \%$ of cases) of thrombosis is plaque rupture ${ }^{163}$ and the remaining cases are predominantly the result of plaque erosion (the latter being much more frequent in young individuals, particularly women) (FIG. 4). A minority (typically around $5 \%$ ) are due to thrombosis forming on calcified nodules. However, thrombosis and clinical sequalae are not an inevitable consequence of atherosclerosis; analysis of autopsies has shown that plaques often show evidence of silent (non-occlusive) thrombi which have undergone repair and healing. Furthermore, the widespread uptake of clinical interventions, including lipid-lowering, are changing the clinical presentation of atherosclerosis in association with changes in the characteristics of the 'vulnerable plaque'164.

As the fibroatheroma develops, so does the necrotic core; the free cholesterol content and calcification increases, and there is breakdown and remodelling of the fibrous cap ECM. The latter is thought to be principally due to the actions of proteases (in particular metalloproteinases ${ }^{165}$ ), but also by sulphatases and exoglycosidases that are predominantly released by macrophages ${ }^{166}$, but may also come from VSMCs ${ }^{167}$. Concomitantly, VSMCs are depleted through cell death, and so the cap diminishes, whilst the growing necrotic core extends outwards, leading to thinning of the fibrous cap ${ }^{168,169}$. Thin-cap fibroatheromas 
(TCFA) are defined by a fibrous cap of less than $65 \mu \mathrm{m}$, and are also known as 'vulnerable plaques' because studies have shown that these plaques are highly susceptible to rupture. The underlying mechanisms are ill-defined, but proteolytic activity ${ }^{166,167}$, mechanical stress ${ }^{170}$ and micro-calcification of the fibrous cap ${ }^{149,171}$ have all been linked to plaque rupture.

Plaque rupture is inversely correlated with VSMC number ${ }^{142}$, which is determined by proliferation, migration and death of VSMCs. Advanced human lesions show little VSMC proliferation $^{172,173}$, but VSMC death, through apoptosis and necrosis (Box 4), is increased compared to normal vessels ${ }^{174-176}$, and in unstable versus stable plaques ${ }^{177}$. Indeed, VSMC apoptosis has been postulated to be key to plaque instability ${ }^{178}$. Seminal work showed plaque VSMCs to spontaneously undergo apoptosis in vitro, with IGF-1 and PDGF acting as survival factors ${ }^{179}$, and plaque VSMCs expressing less IGF-1R ${ }^{180}$. Similarly, cell to cell contact via $\mathrm{N}$-cadherin promotes survival ${ }^{181}$. Conversely, numerous factors that induce VSMC apoptosis have been described, including cell-directed killing (by macrophages, $\mathrm{T}$ lymphocytes and mast cells), ROS, DNA damage, anoikis and cholesterol. Studies of VSMC apoptosis in vivo have utilised mice that have either alterations to apoptotic pathways or systems to induce apoptosis. Early work with adenoviral p53 expression in plaques led to VSMC apoptosis and cap thinning ${ }^{182}$. Similarly, VSMC-specific diphtheria toxin (DT)induced apoptosis revealed short term VSMC apoptosis within established plaques to have no effect on plaque size, but to result in vulnerable plaques with small fibrous caps and a paucity of VSMCs and structural matrix ${ }^{178}$ - a finding subsequently corroborated many times in studies that have promoted or inhibited VSMC death, ${ }^{167,181,183-187}$. Strikingly, DT induction of VSMC apoptosis alongside high fat feeding during atherogenesis resulted in larger plaques $^{51}$, showing that the consequences of VSMC death are more than cell loss alone, and in fact actively drives plaque growth - another well replicated finding ${ }^{167,185,187,188}$. A key controller of VSMC apoptosis in vivo appears to be the survival kinase Akt1 ${ }^{183,186,187}$; conditional ablation of Akt1 during atherogenesis induces VSMC apoptosis and larger plaques, and Akt1 ablation in established plaques leads to a reduced fibrous cap. The contribution of VSMC death to plaque stability is complex and extends beyond direct cell loss; with further consequences on the local milieu (such as initiating calcification ${ }^{150}$ ), and wider effects in activating the immune system. The plaque environment is known to inhibit phagocytosis ${ }^{133-136}$, and defective efferocytosis of apoptotic cells leading to secondary necrosis and leakage of intracellular contents has been proposed to exacerbate the inflammatory milieu ${ }^{131,132,137}$. Indeed, necrotic VSMCs potently drive inflammation via IL$1 \alpha$ due to a lack of IL-1R2 that normally binds and inhibits IL-1 $\alpha^{133,189}$. Thus, a consensus appears whereby functional VSMCs are essential to maintain the fibrous cap and thus plaque stability, but death of VSMCs is a potent driver of atherogenesis.

A recent study of the VSMC transcriptome in symptomatic versus asymptomatic carotid plaques has also highlighted the importance of VSMC senescence ${ }^{190}$. Unstable mature plaques show low VSMC proliferation and clear evidence of VSMC senescence ${ }^{191}$. Senescent VSMCs were originally thought to promote plaque instability through inaction i.e. a lack of VSMC proliferation and matrix production leads to weakening of the fibrous cap. However, senescent VSMCs establish a robust IL- $1 \alpha$-driven SASP containing multiple inflammatory cytokines, chemokines, MMPs and osteogenic factors ${ }^{80,192}$. Thus, the VSMC SASP can recruit mononuclear cells, induce endothelial cell adhesion receptor expression and activate adjacent normal VSMCs ${ }^{80}$, effectively amplifying the effect of a small number of senescent VSMCs. Senescent VSMCs also produce less collagen and release active MMP9 $^{80}$, while BMP2 and osteoprotegerin within the SASP drive calcification ${ }^{192}$. Thus, 
senescent VSMCs can have a negative impact on plaques through both loss of normal function and a direct effect on the local plaque milieu.

An alternative route to thrombosis and clinical sequalae is through plaque erosion. Erosion refers to the formation of a thrombus in the absence of rupture at sites of endothelial denudation or disruption. The underlying plaque may be an intimal thickening or fibroatheroma $^{88,169}$, but VSMCs are often abundant, amidst a proteoglycan-rich ECM, enriched in type III collagen, versican and hyaluronan ${ }^{193}$. Recent studies have identified an important role for hyaluronan, which activates TLR2 signalling upon degradation ${ }^{194}$ and this combined with altered shear stress, leads to endothelial cell activation and apoptosis ${ }^{195}$, neutrophil recruitment and thrombosis ${ }^{194}$. Thus VSMCs are implicated in the events leading to plaque erosion, in particular as the major source of hyaluronan ${ }^{196}$.

\section{Future perspectives}

\section{Difficulties in extrapolating studies from mice to man}

Reconciling the results of studies of animal models with those of human atherosclerosis can be challenging, as there are some important differences in how the disease progresses in humans and animal models. This is exemplified in the case of DITs, which are absent in most animal models. Another fundamental difference is that fibroatheromas rarely progress to rupture in animal models, exemplified by the recently reported effects of a neutralising IL$1 \beta$ antibody, which were deleterious on the fibrous cap in mice ${ }^{141}$, but beneficial in reducing cardiovascular events in the CANTOS trial in humans ${ }^{197}$. Nonetheless, animal models have been instructive in delineating important pathways and basic principles that might underlie plaque development in humans. This is particularly true of the lineage tracing studies in mouse models of atherosclerosis, which have unambiguously established the importance of clonality and phenotype switching of VSMCs. Combinatorial genetic depletion models will likely be instrumental in assessing whether biasing the phenotype of VSMC-derived cells could be a potential treatment avenue. Recently developed techniques, including mass cytometry (СуToF) and single-cell omics (genomics, transcriptomics and epigenomics), hold great promise for high resolution, spatio-temporal analysis of plaque cells in situ, and are likely to provide the conclusive human counterpart and mechanistic data for the aforementioned studies.

\section{VSMCs and genetics of atherosclerosis}

Over 150 CAD loci have been identified from GWAS and other genetic association studies $^{198}$, many of which are associated with disease independently of other known risk factors. Thus, elucidation of the underlying molecular mechanisms may reveal novel pathways and hence targets for therapeutic intervention. However, identification of causal variants is usually far from trivial; CAD loci are often located in non-coding regions, where the causal variant is predicted to effect regulation of gene expression, which may operate over large distances and be cell-type or context specific. Studies are ongoing to identify and functionally characterise the causal variants responsible for each of the CAD loci, and in vitro studies of VSMCs are proving an invaluable resource in this quest. Integration of transcriptomic and epigenomic maps from VSMCs (and other plaque cells) with those of the 
genetic architecture of CAD can be very informative for prioritising variants (and potential pathways) for functional characterisation ${ }^{199,200}$. Unsurprisingly, given the key role of VSMCs in atherosclerosis, a number of loci have been predicted to modulate disease risk through mechanisms specific to VSMCs ${ }^{200}$. Thus, studies in cultured VSMCs, and more recently VSMCs derived from stem cells ${ }^{49,201}$, are likely to be instrumental in the functional characterisation of CAD variants. Recent pioneering examples of such studies include the characterisation of the SMAD3 and TCF21 loci ${ }^{202}$.

\section{Conclusion}

The role of VSMCs in atherosclerosis extends far beyond that perceived for decades. VSMCs and VSMC-derived cells comprise a (if not the) major source of plaque cells, and contribute to numerous plaque cell phenotypes, including macrophage-like and foam cells, in addition to cells responsible for producing the atherogenic and or athero-protective ECM throughout the disease. Thus, VSMCs are implicated mechanistically at all stages of atherosclerosis, and recent studies have established the extent and importance of VSMC clonality and phenotype switching in plaque progression. These concepts have been around for decades, but it is only very recently that technologies for genetic engineering and imaging have converged with a deeper understanding of developmental processes to generate conclusive data in animal models. The era of single cell omics promises to deliver the evidence as to if and how these processes contribute to the disease in humans. It is clear that a better understanding of the biology of VSMCs is required if we are to fulfil aspirations of selectively targeting 'culprit' cells or manipulating cell phenotype to enhance clinical benefit and/or avert processes that are detrimental in disease.

\section{Key points:}

- VSMCs and VSMC-derived cells are a major source of plaque cells and ECM at all stages of atherosclerosis

- VSMCs contribute to many different plaque cell phenotypes, including ECM-producing cells of the fibrous cap, macrophage-like cells, foam cells, mesenchymal stem cell-like and osteochondrogenic cells

- Recently progress has been made regarding the source of plaque VSMCs and VSMCderived cells, which highlights the importance of developmental origin, clonal expansion and phenotype switching of VSMCs in atherosclerosis 


\section{Box 1: Historical perspective on VSMCs in atherosclerosis}

The development of antibodies for 'VSMC-specific' function-associated markers, such as smooth muscle alpha actin ( $\alpha \mathrm{SMA})^{6-9}$, greatly facilitated immuno-histological studies of VSMCs in plaques of animal models ${ }^{203,204}$ and humans ${ }^{98,103}$. These studies, alongside in vitro culture models ${ }^{55}$ and models of arterial injury, such as balloon angioplasty, revealed that VSMCs are capable of great phenotypic plasticity, and undergo 'phenotypic switching' from contractile to proliferative synthetic phenotypes ${ }^{205-207}$. Phenotype switching and proliferation of VSMCs in response to arterial injury and lipid infiltration were considered the main pathological processes underlying plaque development ${ }^{207}$.

Studies in the 1990s characterised the role of VSMC proliferation, migration, apoptosis, and phenotype switching in atherogenesis ${ }^{208}$, and revealed that VSMCs can give rise to foam cells $^{4,5,102}$ and osteochondrogenic cells ${ }^{154}$. However, detailed post-mortem analyses of culprit plaques in sudden cardiac death established that the integrity of the fibrous cap, comprising mostly $\alpha$ SMA-positive cells and associated extracellular matrix (ECM), is critical to stabilise and protect plaques from rupture, a major cause of the clinical sequalae of atherosclerosis $^{142,163,168}$. These studies also highlighted the role of immune cells, particularly macrophages, and inflammation as the main driver of plaque development ${ }^{169}$. Thus, the prevailing model has been that VSMCs contribute to the cellularity and inflammation of the developing plaque, but have a predominantly beneficial role in its stabilisation though elaborating the fibrous cap ${ }^{209}$.

In the last decade, studies applying fate mapping and lineage tracing techniques have revealed the limitations of relying on 'VSMC-specific' function-associated markers to infer VSMC identity, and exposed the extent to which this can lead to false negative and false positive identification of VSMCs, as well as oversimplification of VSMC heterogeneity and functions in plaques ${ }^{11,17,18}$.

\section{Text boxes (for timeline):}

pre 1900s histology on morbid specimens, including by Virchow (1856) who proposed atherosclerosis to result from inflammation and proliferation as a consequence of arterial injury by mechanical forces

\section{Marchand coins 'atherosclerosis'}

Ignatowsky describes relationship between protein/lipid-rich diet and experimental atherosclerosis, these studies were extended by Anichkov in 1913, who discovered the importance of cholesterol

Foam cells observed in human and experimental atherosclerosis studies by light microscopy 210,211

Pease describes VSMC as the only cell-type in the healthy media by electron microscopy ${ }^{2}$. Studies of experimental and human atherosclerosis quickly followed, revealing VSMC derived cells as prominent cell type in plaques ${ }^{3-5}$ 
1 Wissler proposes VSMC are the primary cell type involved in atherosclerosis, assimilating

2 many studies (including Wolinsky \& Glagov ${ }^{212}$ ) that VSMC are the contractile and ECM-

3 producing cells of the media and, furthermore, contribute to plaque foam cells ${ }^{62}$

4

5

6

7

8

9

10

11

12

13

14

15

16

17

18

19

20

21

22

23

24

25

26

27

28

29

30

31

32

33

34

35

36

37

38

39
Ross further develops 'response to injury hypothesis', emphasizing the role of PDGF

mediated VSMC proliferation ${ }^{207}$ (firstly due to EC injury and platelet activation ${ }^{213}$ and later updated to incorporate a role for macrophage derived PDGF ${ }^{129}$ )

Benditt \& Benditt propose plaque VSMC arise from clonal expansion ${ }^{46}$

Chamley-Campbell et al identify phenotype switching in cultured VSMCs ${ }^{55}$

'vulnerable plaque' concept developed; studies of culprit plaques in cardiac deaths identify fibrous cap integrity essential to plaque stability ${ }^{163,168,214}$

ApoE and LDLR mouse models of atherosclerosis developed 203,204

'response to retention hypothesis' proposed ${ }^{113}$ and supported by identification of the central role of ApoB containing lipoproteins ${ }^{101}$

first lineage tracing studies ${ }^{11,17,18}$ which collectively revealed VSMC contribution much more substantial than previously thought, giving rise to macrophage marker positive cells, foam cells, osteochondrogenic and mesenchymal stem cell like cells

multi-colour lineage tracing studies demonstrate multiple plaque phenotypes are derived from common ancestor - revealing the true extent of VSMC clonality in plaques ${ }^{20,22}$

CANTOS trial establishes causal role for inflammation in pathogenesis of atherosclerosis ${ }^{197}$ 


\section{Box 2: Embryonic origins of VSMCs and sources of VSMC progenitors in adults}

During embryonic development, medial VSMCs (and in some instances pericytes ${ }^{215}$ ) arise from local progenitor cells, of which there are multiples distinct lineages distributed across the arterial tree. In mice, more than eight distinct progenitor populations have been identified $^{44,216,217}$. The aortic root and outer medial layers of the ascending aorta derive from the secondary heart field ${ }^{26,28}$; the inner medial layer of the ascending aorta, aortic arch, ductus arteriosus, innominate and right subclavian arteries, right and left common carotid arteries derive from the neural crest ${ }^{25}$; the descending aorta derives from paraxial (somatic) mesoderm ${ }^{218}$; and the coronary arteries are derived from pro-epicardium, which derives from lateral plate mesoderm ${ }^{219}$.

Potential VSMC progenitor populations have also been identified in the media in the adult mouse, including VSMC-derived cells expressing Sca1 and other mesenchymal stem cell markers ${ }^{11,43}$. These cells may be an intermediate population derived from phenotypic switching, which can give rise to different VSMC-derived cell phenotypes ${ }^{43}$. Other potential progenitor cells include a population of adventitial cells located close to the medial boundary that express mesenchymal stem cell markers (e.g. Sca1) and are sonic hedgehog signallingresponsive (Gli1 positive) ${ }^{27,220-222}$, and pericytes ${ }^{223,224}$, which are VSMC-like cells of the microvasculature.

Importantly, studies have shown that progenitors with distinct origins may achieve a common VSMC fate with respect to expression of 'VSMC-specific' function-associated markers (through pathways discussed in Box 3), but are nonetheless distinct with respect to other functional characteristics, such as responses to growth factors. 


\section{Box 3: Molecular mechanisms underlying VSMC plasticity \\ Transcription factors:}

Myocardin (MYOCD) family proteins drive expression of contractile genes ${ }^{57}$. MYOCD is a co-factor for serum response factor (SRF), which binds CArG-box elements within contractile gene promoters. Most environmental cues and signalling pathways affecting VSMC function impact the expression and/or activity of MYOCD 225,226

KLF4 represses contractile gene expression through several mechanisms, including binding to $\mathrm{G} / \mathrm{C}$ repressor elements and inhibiting SRF binding to CArG-boxes. KLF4 inhibits proliferation; VSMC specific deletion of CHOP leads to decreased VSMC proliferation through increased expression of KLF4 ${ }^{227}$. Importantly, VSMC phenotype switching is KLF4 dependent. KLF4 is required for induction of progenitor cells prior to clonal expansion of pulmonary VSMCs in hypoxia ${ }^{65,66}$ and VSMC-specific deletion of KLF4 in ApoE-/- animals results in reduced numbers of VSMC-derived macrophage and mesenchymal stem cell marker positive plaque cells ${ }^{11}$.

Extracellular stimuli: the contractile phenotype is promoted by TGF- $\beta$, whereas PDGF induces KLF4 expression, VSMC proliferation and phenotypic switching. Other growth factors including WNT signalling also promote proliferation and migration of VSMCs. Proinflammatory cytokines (e.g. IL-1 and TNF- $\alpha$ ) perturb VSMC phenotype via NF- $\kappa$ B and AP1 mediated gene regulation, including MYOCD downregulation. Cholesterol-induced activation of macrophage-associated gene expression in VSMC occurs via microRNA$143 / 145$, involves MYOCD and inflammatory signalling and is affected by KLF4 ${ }^{59,228}$.

Cell interactions: ECM proteins and heparin affect VSMC phenotype ${ }^{229}$. Notably, deletion of integrin $\beta 3$ results in larger lesions and affects VSMC clonality in atherosclerosis ${ }^{23}$. Differences in how cells communicate with the environment may also explain the documented effect of stretch and shear stress on VSMC phenotype ${ }^{230}$.

Epigenetic regulation: the reversibility of VSMC phenotypic switching indicates a cellular memory of the contractile state. Indeed, contractile genes remain marked by H3K4me2 (generally associated with actively transcribed genes) after phenotypic switching ${ }^{18}$ and manipulation of DNA methylation and histone modifying enzymes directly affect VSMC behaviour in murine models of vascular injury and atherosclerosis ${ }^{231-233}$, whilst levels of epigenetic markers are altered in human plaques ${ }^{234}$. Non-coding RNAs also control VSMC plasticity ${ }^{235,236}$ evidenced by the effect of specific miRNAs and long non-coding RNAs on VSMC biology and function ${ }^{237,238}$. 


\section{Box 4: Mechanisms of cell death}

Apoptosis: the commonest form of programmed cell death (PCD) utilised throughout development and day-to-day physiology. Executed by apoptotic caspases (e.g. 3, 7), with main initiation pathways controlled via the mitochondria (via Bcl-2 family members) or external death receptors (e.g. Fas, TNFR). Apoptotic cells must be phagocytosed, or secondary necrosis with leakage of inflammatory contents (including DAMPs) will occur. All major cell types within the plaque are witnessed to undergo apoptosis.

Autophagic cell death: a mechanism for the organised degradation and recycling of intracellular components within double membraned autophagosomes that fuse with lysosomes. Can be a response to stress that enables the cell to survive, but is also witnessed as PCD. VSMC specific deficiency in autophagy leads to increased VSMC death and enhanced features of vulnerable plaques ${ }^{188}$.

Necrosis: An un-programmed form of cell death characterized by catastrophic loss of plasma membrane integrity and leakage of cell contents. Uncleared dying cells default to secondary necrosis. Difficult to prove in vivo, but ultrastructural evidence suggests necrotic plaque macrophages and VSMCs occur.

Necroptosis: A programmed form of necrosis allowing cell suicide when apoptosis is blocked (e.g. viral caspase inhibitors). Utilises RIPK1/3 to form the ripoptosome which activates MLKL that destroys the plasma membrane. Increased RIP3 and MLKL reported in human plaques, but difficult to specifically detect necroptosis.

Pyroptosis: Inflammatory form of cell death that occurs in concert with inflammasome activation and IL-1 production, often in response to intracellular infection. Leads to activation of inflammatory caspases (e.g. 1, 4, 5, 11) that activate IL-1 and/or the poreforming protein GSDMD, and subsequent membrane permeabilisation. Likely happens in plaques after cholesterol crystal activation of macrophage NLRP3 inflammasomes.

Paraptosis: caspase-independent cell death leading to cytoplasmic vacuolation and eventual osmotic lysis. Not currently described in atherosclerotic plaques. 
Table 1: Lineage tracing studies in atherosclerosis

\begin{tabular}{|c|c|c|c|c|c|c|c|c|c|}
\hline $\begin{array}{l}\text { Cell type } \\
\text { studied }\end{array}$ & Cell tracing* & Mouse model & $\begin{array}{l}\text { contribution of labelled } \\
\text { cells to plaque? }\end{array}$ & vsmCs & aSMA negative? & Macrophage-like & Osteochondrogenic & MSC-like & Ref \\
\hline VSMC & \begin{tabular}{|l|} 
Tagin-CreERt2/R26R-LacZ or R26R- \\
mT/mG or R26R-Confetti
\end{tabular} & $\begin{array}{l}\text { ApoE-I- chow (52 weeks) or HFD } \\
\text { (16 weeks) }\end{array}$ & yes, clonal patches & aSMA+ & Yes & $\begin{array}{l}\text { Lgals33, CD68+ (62,54\% } \\
\text { respectively of labelled } \\
\text { cells) }\end{array}$ & $\mathrm{NA}$ & NA & 17 \\
\hline VSMC & Myh11-CreERt2/R26R-EYFP & ApoE-l-HFJ 18 weeks & yes & aSMAt & $>95 \%$ of labelled cells & NA & $\mathrm{NA}$ & NA & 18 \\
\hline VSMC & Myh11-CreERt2/R26R-EYFP & ApoE-I- HFJ 18 weeks & yes & $\begin{array}{l}16 \% \text { of labeled cells } \\
\text { aSMA+ }\end{array}$ & \begin{tabular}{|l}
$12 \%$ of labelled cells \\
Pdgfbrt, $32-51 \%$ of \\
labelled cells unknown \\
identity
\end{tabular} & $\begin{array}{l}30 \% \text { of labelled cells } \\
\text { Lgals } 3+\end{array}$ & NA & 7\% of labelled cells Sca $1+$ & 11 \\
\hline VSMC & Myh11-CreERt2/R26R-Confetti & ApoE-/- HFD $16-19$ weeks & \begin{tabular}{|l}
$70(40-90) \%$ of plaque \\
cells, oligoclonal
\end{tabular} & $\begin{array}{l}30-100 \% \text { of labelled cells } \\
\text { aSMA+, 70-100\% of } \\
\text { aSMA+ cells labelled } \\
\end{array}$ & yes & $\begin{array}{l}5-50 \% \text { of labelled cells } \\
\text { Lamp2+, } 70 \% \text { of Lamp2+ } \\
\text { cells were labelled }\end{array}$ & NA & NA & 20 \\
\hline VSMC & Myh11-CreERt2/R26R-Confetti & \begin{tabular}{|l}
$\begin{array}{l}\text { PCSKG9-D377Y AAV, 12-36 week } \\
\text { HFD }\end{array}$ \\
\end{tabular} & \begin{tabular}{|l|}
$\begin{array}{l}\text { oligo clonal VSMC } \\
\text { contribution to plaque cap } \\
\text { and core }\end{array}$ \\
\end{tabular} & aSMAt & yes & \begin{tabular}{|l}
$\begin{array}{l}\text { Oil Red O+, no Lgals3+ } \\
\text { cells detected }\end{array}$ \\
\end{tabular} & yes & yes & 22 \\
\hline VSMC & Myh11-CreERt2/Brainbow & ApoE-I- HFD 5-12 weeks & \begin{tabular}{|l|}
$\begin{array}{l}\text { monoclonal VSMC } \\
\text { contribution to plaque cap } \\
\text { and core }\end{array}$ \\
\end{tabular} & aSMAt & yes & NA & NA & NA & 23 \\
\hline VSMC & Myh11-CreERt2/R26R-Confetti & ApoE-l-HFD 16-19 weeks & yes, clonal patches & aSMAt & yes & yes & Sca1+ (rare) & Sca1+(rare) & 43 \\
\hline Unknown & Chimeras & ApoE-I- Chow diet 10 months & $\begin{array}{l}\text { ligoclonal patches in plaqe } \\
\text { cap }\end{array}$ & clonal aSMA+ & NA & NA & NA & NA & 22 \\
\hline $\begin{array}{l}\text { Tof21+ } \\
\text { (Adventitial) }\end{array}$ & TCF21-MerCreMer/R26R-tdTomato & $\begin{array}{l}\text { ApoE-l- HDF } 12 \text { weeks, Ldll-l- HFD } \\
16-20 \text { weeks }\end{array}$ & yes & Tagint+ & Periostin+ & NA & $\mathrm{NA}$ & NA & 239 \\
\hline $\begin{array}{l}\text { Adventitial } \\
\text { cells }\end{array}$ & \begin{tabular}{|l|} 
transplant of cultured Sca-1+ \\
adventitial cells from SM-LacZ/ApoE-I- \\
donor animials into ApoE-l- hosts
\end{tabular} & vein graft & yes & LacZ+ cells in plaque & NA & NA & NA & NA & 220 \\
\hline $\begin{array}{l}\text { Adventitial } \\
\text { MSC }\end{array}$ & Gli1-CreERt2/R26R-tdTomato & $\begin{array}{l}\text { ApoE-/- with subtotal (5/6) } \\
\text { nephrectomy and HFD } 10-16 \text { weeks }\end{array}$ & $\begin{array}{l}\text { observed (40 cells/high } \\
\text { power field) }\end{array}$ & $\begin{array}{l}\text { Calponin }+(20-80 \% \text { of } \\
\text { lineage traced cells })\end{array}$ & yes & no $\mathrm{CD} 68+$ cells detected & \begin{tabular}{|l} 
calcium tracert, Run $\times 2+$ \\
$\left(\begin{array}{l}(10-25 \% \text { of lineage traced } \\
\text { cells) }\end{array}\right.$
\end{tabular} & 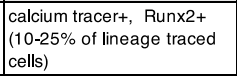 & 221 \\
\hline BM-derived & $\begin{array}{l}\text { BM from GFP+ donor animals } \\
\text { transplanted into GFP- hosts }\end{array}$ & ApoE-I- HFD 20-32 weeks & \begin{tabular}{|l|}
$\begin{array}{l}\text { Mac2+ foam cells in plaque } \\
\text { core }\end{array}$ \\
\end{tabular} & No & yes & Lgals3+ & NA & NA & 41 \\
\hline BM-derived & $\begin{array}{l}\text { BM from GFP+ donor animals } \\
\text { transplanted into GFP- hosts }\end{array}$ & $\begin{array}{l}\text { Healing plaque (ApoE--l with } \\
\text { spontaneous or mechanically } \\
\text { disrupted hemornagic plaque) }\end{array}$ & \begin{tabular}{|l|} 
Mac2+ foam cells in plaque \\
core
\end{tabular} & No & yes & Lgals3+ & NA & $\mathrm{NA}$ & 40 \\
\hline BM-derived & $\begin{array}{l}\text { BM from MYH11-Cre/R26R- } \\
\text { LacZ/ApoE-I- donor animals } \\
\text { transplanted into ApoE---hosts }\end{array}$ & ApoE-I- HFD 6-22 weeks & $\begin{array}{l}0.7 \% \text { of cells in advanced } \\
\text { plaque were LacZ+ }\end{array}$ & $\begin{array}{l}\text { very rare }(0.4 \% \text { of plaque } \\
\text { cells were aSMA+LacZ }+)\end{array}$ & very rare & NA & NA & NA & 42 \\
\hline EC & end.ScICreERT/R26R-EYFP & ApoE-I- HFD 8-30 weeks & yes & $\begin{array}{l}\text { Yes, low contribution } \\
\text { (asMA/SMMHC) }\end{array}$ & $\begin{array}{l}\text { yes, } 32-45 \% \text { of FAP+ } \\
\text { fibroblasts are labelled }\end{array}$ & NA & NA & $\mathrm{NA}$ & 240 \\
\hline ND & not detected & & & & & & & & \\
\hline NA & not analysed & & & & & & & & \\
\hline HFD & high fat diet & th indution th o th & & & & & & & \\
\hline R26R- & $\begin{array}{l}\text { tamoxixen-indiced recombin } \\
\text { ROA } 26 \text { locus reporter - }\end{array}$ & to induction of atherosclerosis & & & & & & & \\
\hline
\end{tabular}


Fig 1

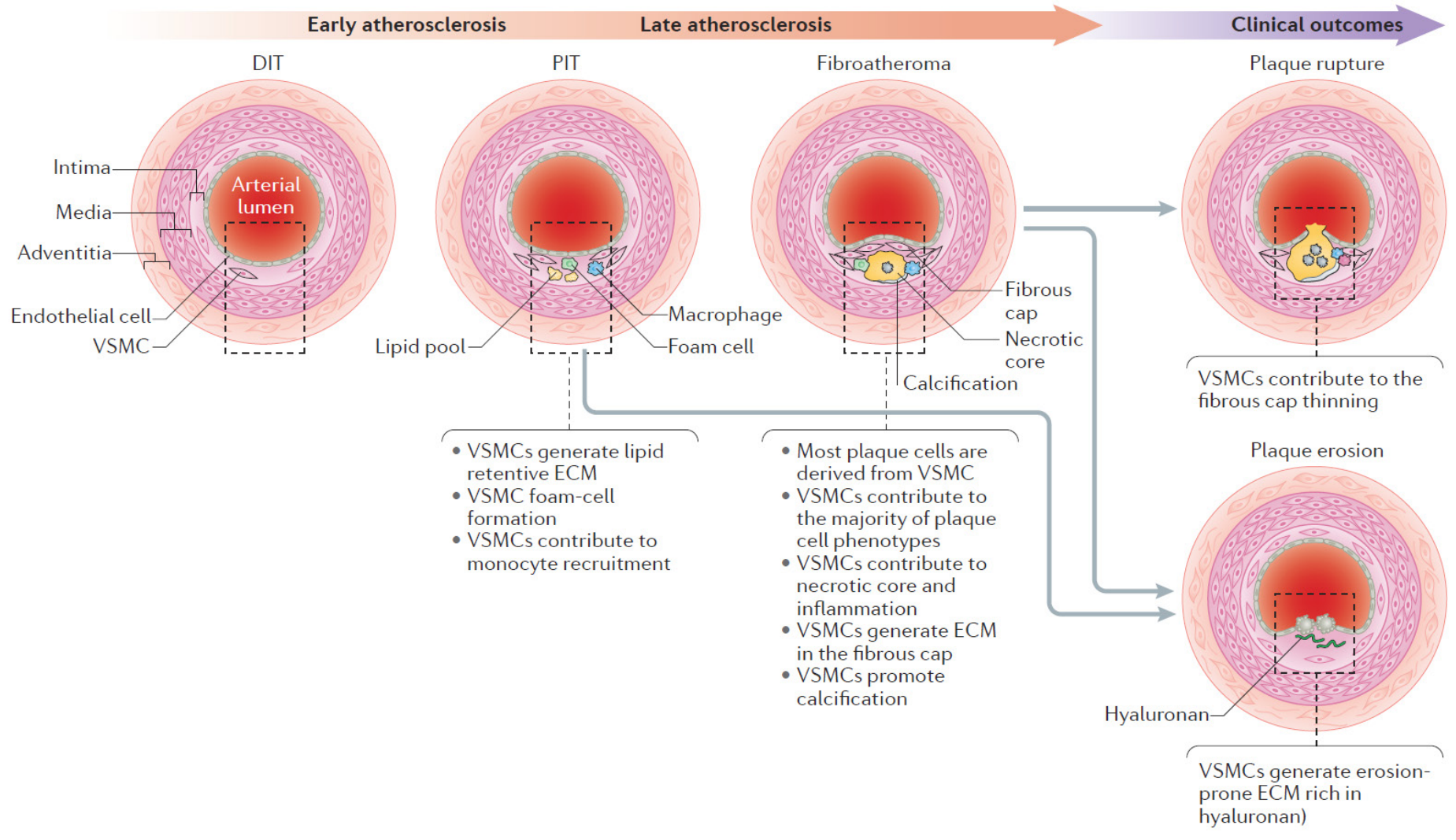


Fig 2

Healthy artery
Arterial- Non-thickened artery
lumen

\section{Early atherosclerosis}

\section{DIT}

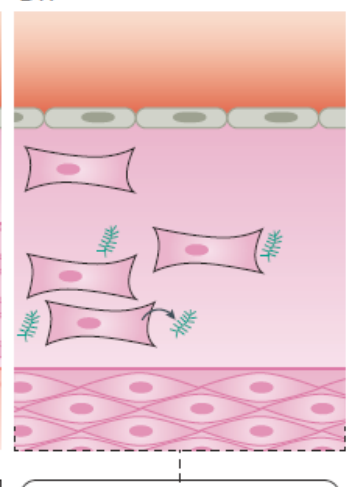

Phenotype switching

- VSMCs are synthetic ( $\downarrow$ MYOCD)

- Synthetic VSMCs

elaborate ECM rich in proteoglycans (biglycan, versican and perlecan) and glycosaminoglycans (hyaluronan), and have lower levels of contractile proteins

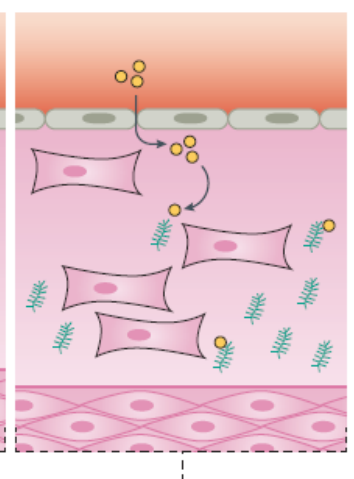

\section{Lipid retention}

- Plasma-derived LDL is retained via interactions between positively charged apolipoproteins (particularly ApoB) and negatively charged side chains of VSMCderived proteoglycans (chondroitin sulfate in biglycan and versican, and heparin sulfate in perlecan)

- Retained LDL is more susceptible to oxidation

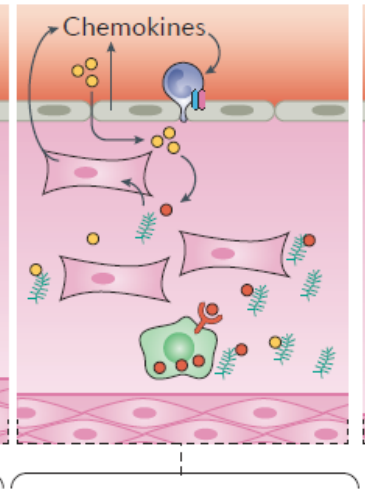

Recruitment of monocytes

- Activated VSMCs (and EC) secrete chemokines (CCL2, CCL5 and CXCL1) that, together with OxLDL, attract monocytes to the inflamed endothelium, which expresses adhesion molecules (selectins, ICAM1, VCAM1 and CD31)

\section{Foam-cell formation}

- $\uparrow$ SR levels and $\downarrow$ levels of cholesterol esterase and cholesterol efflux transporter (ABCA1) in VSMCs, leading to oxLDL uptake and foam cell formation
PIT

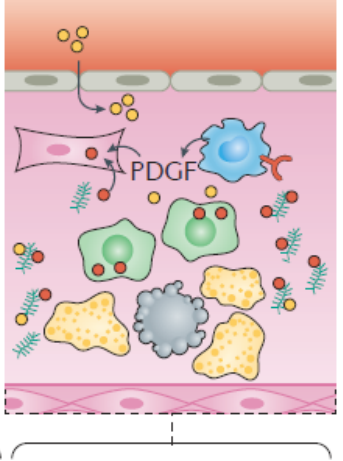

Apoptosis

- OxLDL uptake leads to VSMC apoptosis

Phenotype switching

- VSMCs lose expression of genes encoding contractile proteins (such as alphaSMA) and express markers of other cell types (such as CD68) 


\section{Late atherosclerosis}

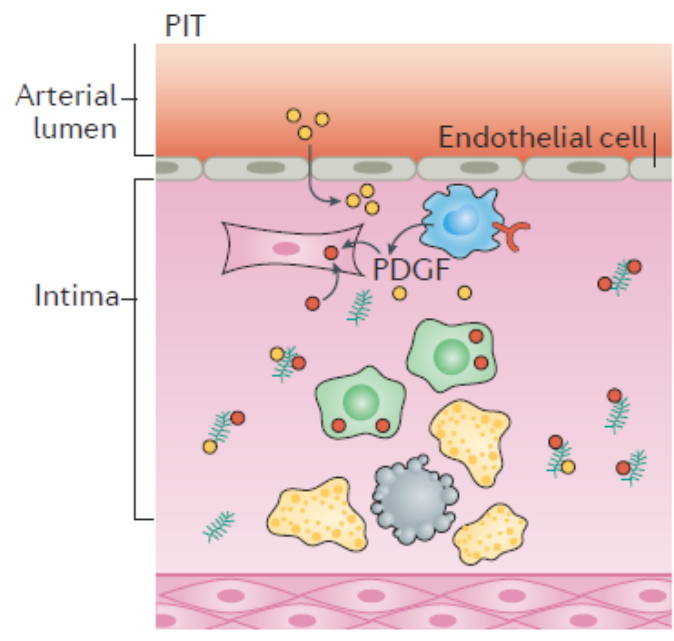

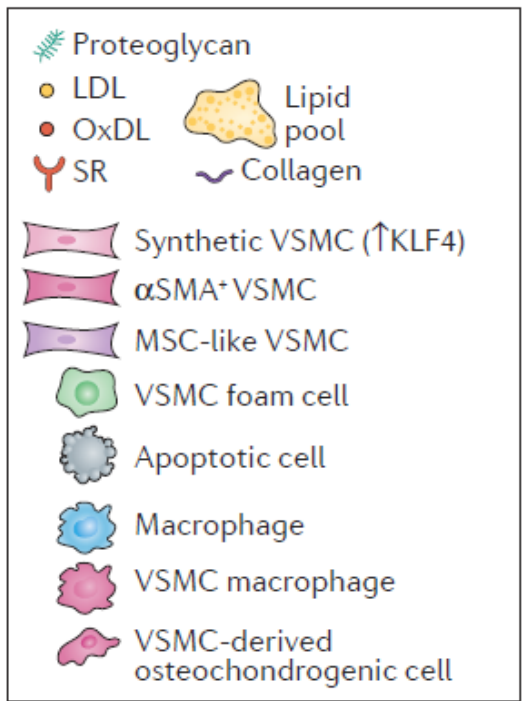

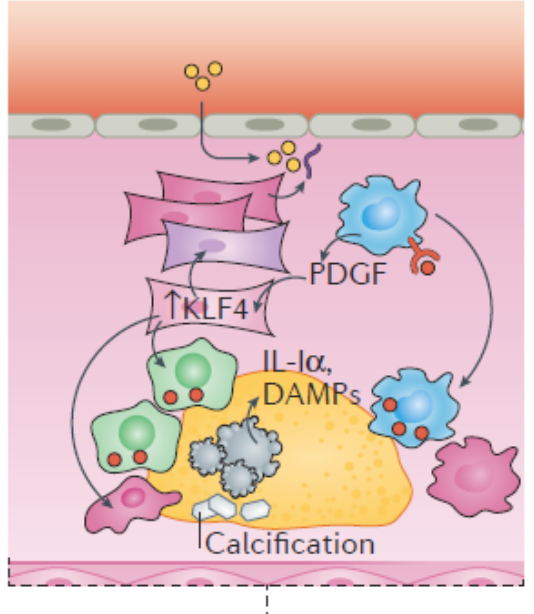

\section{Phenotype switching}

- Lipid loading, $\downarrow$ ECM contacts and $\uparrow$ inflammation lead to altered VSMC phenotypes ( $\uparrow \mathrm{KLF} 4$ ), including aSMA+ cells, macrophage-like cells, foam cells, MSC-like cells and osteochondrogenic cells

\section{Necrotic core formation}

- $\uparrow$ Apoptosis and suppressed efferocytosis lead to secondary necrosis and inflammation via release of DAMPs

\section{Calcification}

- Calcifying microvesicles (from apoptotic and osteochondrogenic cells) lead to deposition of large calcification granules
Fibroatheroma

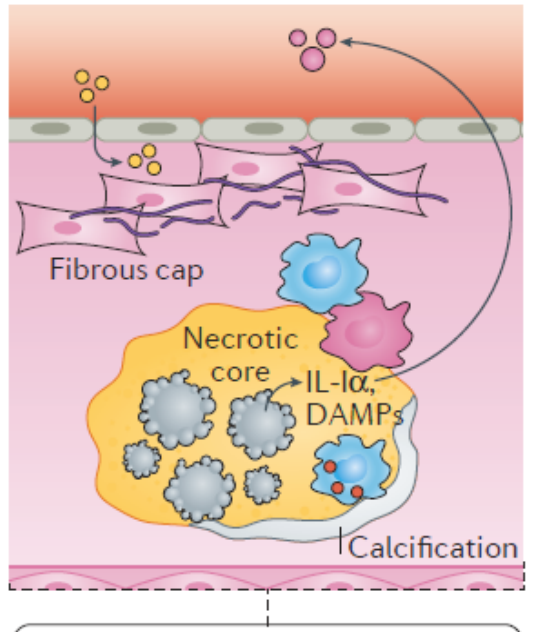

\section{Fibrous cap formation}

- VSMC proliferate and generate altered ECM ( $\downarrow$ proteoglycans and $\uparrow$ collagens)

\section{Enlarged necrotic core}

- Apoptosis and suppressed efferocytosis continue to lead to secondary necrosis and inflammation

\section{Calcification}

- Calcium deposits coalesce to form sheets (which can lead to calcium nodules and thrombosis) 


\section{Plaque rupture}

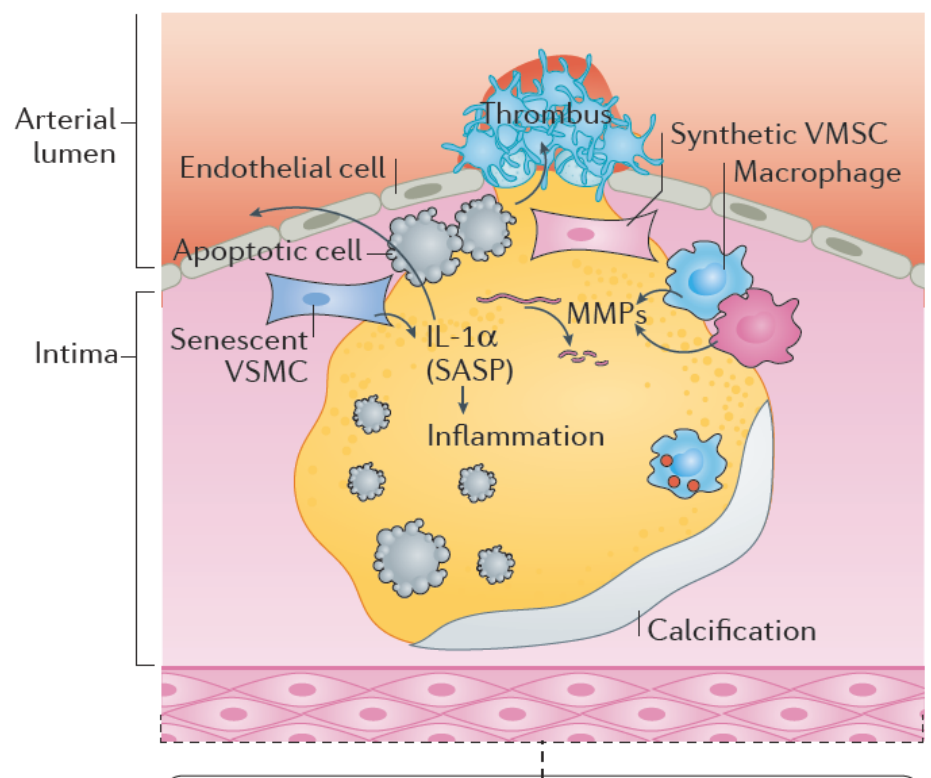

Thinning of the fibrous cap

- VSMC death leads to $\downarrow$ ECM, while macrophages (possibly derived form VSMCs) and VSMCs release MMPs that degrade the ECM

Senescent VSMCs (SASP)

- These cells release IL-1alpha, and pro-inflammatory cytokines (such as IL-6 and IL-8) and MMPs (including MMP9)

\section{Plaque erosion}
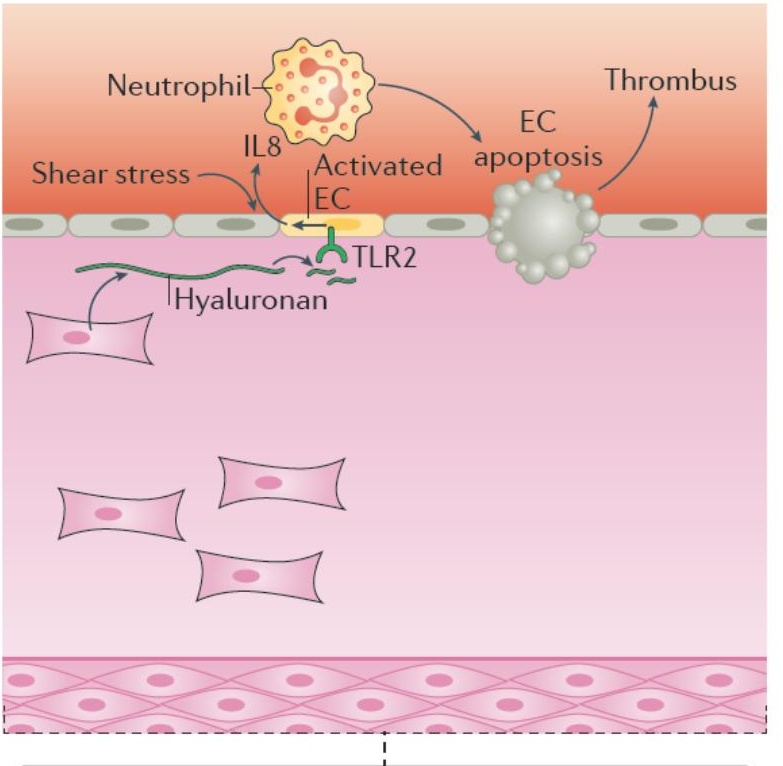

Generation of erosion-prone ECM

- VSMCs produce an altered ECM rich in hyaluronan, type III collagen and versican

- Degraded hyaluronan is a TLR2 ligand, which activates ECs

- Activation of ECs combined with altered shear stress leads to neutrophil recruitment and EC apoptosis, which initiates platelet activation and thrombosis 
Box 2

a

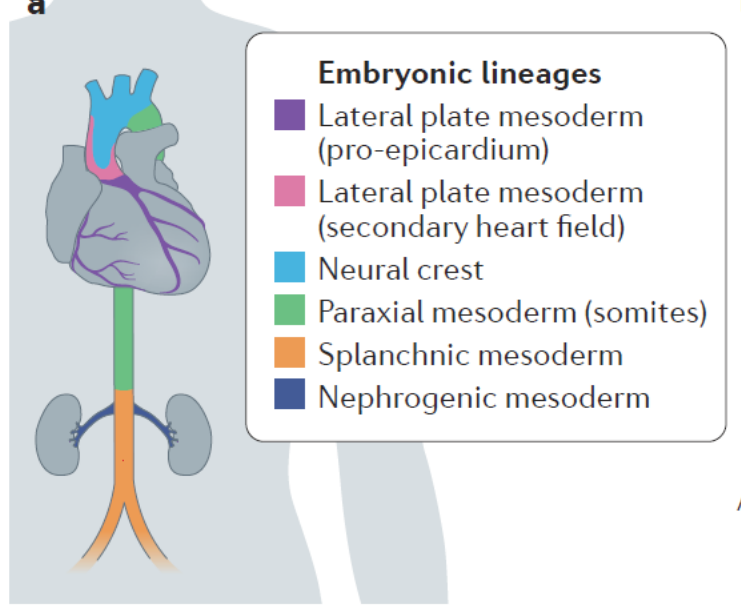

b

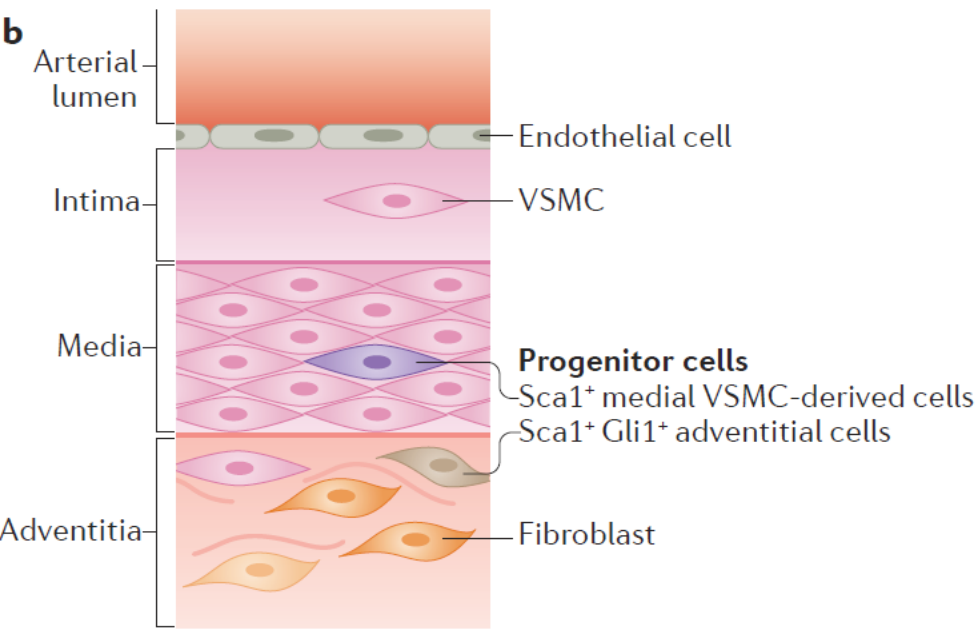

Box 3
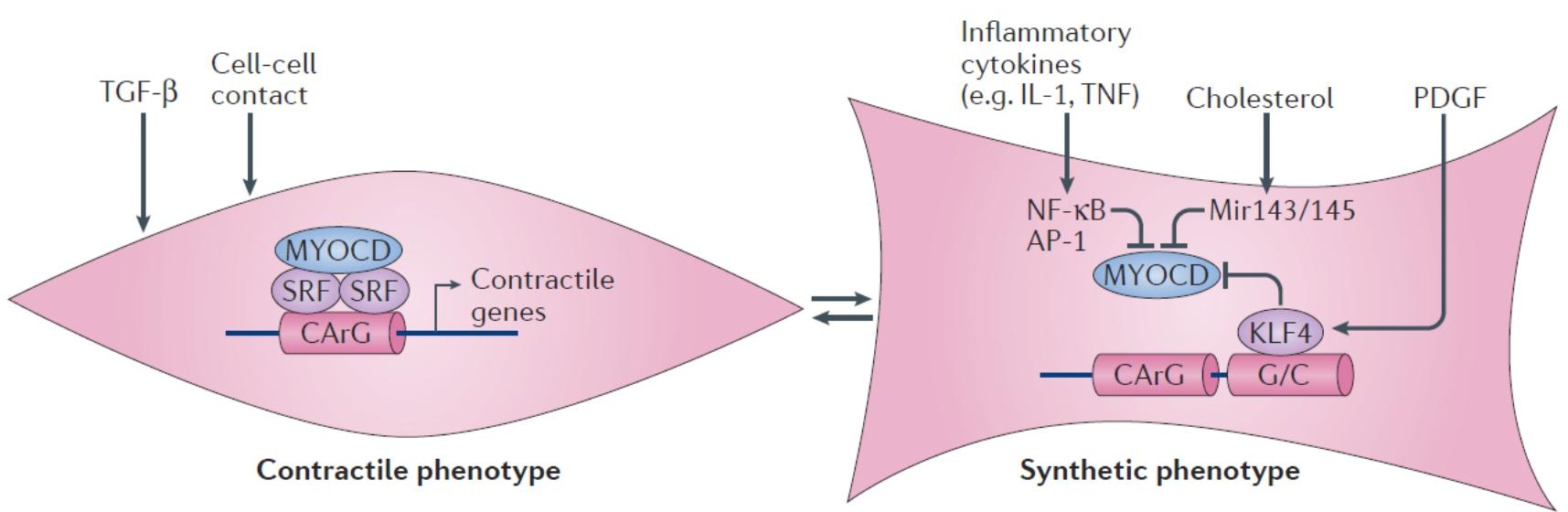


\section{References}

\section{Highlighted references}

Gomez 2013, Feil 2014, Shankman 2015 - these were the first lineage tracing studies of VSMCs in the context of atherosclerosis

Chappell 2016 - this article demonstrates that different VSMC phenotypes arise from the same ancestral cell in atherosclerosis

Misra 2018 - This article provides evidence that secreted factors affect clonality

Childs 2016 - This article demonstrates the impact of senescence in atherosclerosis

1. World Health Organisation (WHO). The top 10 causes of death. Available at: https://www.who.int/news-room/fact-sheets/detail/the-top-10-causes-of-death.

2. Pease, D. C. \& Paule, W. J. Electron microscopy of elastic arteries; the thoracic aorta of the rat. J. Ultrastruct. Res. 3, 469-483 (1960).

3. Parker, F. An Electron Microscopic Study of Experimental Atherosclerosis. Am. J. Pathol. 36, 19-53 (1960).

4. Geer, J. C., McGill, H. C. J. \& Strong, J. P. The fine structure of human atherosclerotic lesions. Am. J. Pathol. 38, 263-287 (1961).

5. Imai, H. et al. Atherosclerosis in rabbits. Architectural and subcellular alterations of smooth muscle cells of aortas in response to hyperlipemia. Exp. Mol. Pathol. 5, 273310 (1966).

6. Chamley, J. H., Groschel-Stewart, U., Campbell, G. R. \& Burnstock, G. Distinction between smooth muscle, fibroblasts and endothelial cells in culture by the use of fluoresceinated antibodies against smooth muscle actin. Cell Tissue Res. 177, 445-457 (1977).

7. Gown, A. M., Vogel, A. M., Gordon, D. \& Lu, P. L. A smooth muscle-specific monoclonal antibody recognizes smooth muscle actin isozymes. J. Cell Biol. 100, 807-813 (1985).

8. Skalli, O. et al. A monoclonal antibody against alpha-smooth muscle actin: a new probe for smooth muscle differentiation. J. Cell Biol. 103, 2787-2796 (1986).

9. Tsukada, T., Tippens, D., Gordon, D., Ross, R. \& Gown, A. M. HHF35, a muscleactin-specific monoclonal antibody. I. Immunocytochemical and biochemical characterization. Am. J. Pathol. 126, 51-60 (1987).

10. Shanahan, C. M. \& Weissberg, P. L. Smooth muscle cell heterogeneity: Patterns of gene expression in vascular smooth muscle cells in vitro and in vivo. Arterioscler. Thromb. Vasc. Biol. 18, 333-338 (1998).

11. Shankman, L. S. et al. KLF4-dependent phenotypic modulation of smooth muscle cells has a key role in atherosclerotic plaque pathogenesis. Nat. Med. 21, 628-637 (2015).

12. Wirth, A. et al. G12-G13-LARG-mediated signaling in vascular smooth muscle is required for salt-induced hypertension. Nat. Med. 14, 64-68 (2008).

13. Kuhbandner, S. et al. Temporally controlled somatic mutagenesis in smooth muscle. Genesis 28, 15-22 (2000).

14. Holtwick, R. et al. Smooth muscle-selective deletion of guanylyl cyclase-A prevents the acute but not chronic effects of ANP on blood pressure. Proc. Natl. Acad. Sci. U. S. A. 99, 7142-7147 (2002).

15. Zhang, J. et al. Generation of an adult smooth muscle cell-targeted Cre recombinase mouse model. Arteriosclerosis, thrombosis, and vascular biology 26, e23-4 (2006).

16. Raja, C. et al. Promoters to Study Vascular Smooth Muscle. Arterioscler. Thromb. 
Vasc. Biol. 39, 603-612 (2019).

17. Feil, S. et al. Transdifferentiation of vascular smooth muscle cells to macrophage-like cells during atherogenesis. Circ. Res. 115, 662-667 (2014).

18. Gomez, D., Shankman, L. S., Nguyen, A. T. \& Owens, G. K. Detection of histone modifications at specific gene loci in single cells in histological sections. Nat. Methods 10, 171-177 (2013).

19. Albarrán-Juárez, J., Kaur, H., Grimm, M., Offermanns, S. \& Wettschureck, N. Lineage tracing of cells involved in atherosclerosis. Atherosclerosis 251, 445-453 (2016).

20. Chappell, J. et al. Extensive Proliferation of a Subset of Differentiated, yet Plastic, Medial Vascular Smooth Muscle Cells Contributes to Neointimal Formation in Mouse Injury and Atherosclerosis Models. Circ. Res. 119, 1313-1323 (2016).

21. Cherepanova, O. A. et al. Activation of the pluripotency factor OCT4 in smooth muscle cells is atheroprotective. Nat. Med. 22, 657-665 (2016).

22. Jacobsen, K. et al. Diverse cellular architecture of atherosclerotic plaque derives from clonal expansion of a few medial SMCs. JCI Insight 2, (2017).

23. Misra, A. et al. Integrin beta3 regulates clonality and fate of smooth muscle-derived atherosclerotic plaque cells. Nat. Commun. 9, 2073 (2018).

24. Nemenoff, R. A. et al. SDF-1alpha induction in mature smooth muscle cells by inactivation of PTEN is a critical mediator of exacerbated injury-induced neointima formation. Arterioscler. Thromb. Vasc. Biol. 31, 1300-1308 (2011).

25. Jiang, X., Rowitch, D. H., Soriano, P., McMahon, A. P. \& Sucov, H. M. Fate of the mammalian cardiac neural crest. Development 127, 1607-1616 (2000).

26. Waldo, K. L. et al. Secondary heart field contributes myocardium and smooth muscle to the arterial pole of the developing heart. Dev. Biol. 281, 78-90 (2005).

27. Passman, J. N. et al. A sonic hedgehog signaling domain in the arterial adventitia supports resident Sca1+ smooth muscle progenitor cells. Proc. Natl. Acad. Sci. U. S. A. 105, 9349-9354 (2008).

28. Sawada, H., Rateri, D. L., Moorleghen, J. J., Majesky, M. W. \& Daugherty, A. Smooth Muscle Cells Derived from Second Heart Field and Cardiac Neural Crest Reside in Spatially Distinct Domains in the Media of the Ascending Aorta - Brief Report. Arterioscler. Thromb. Vasc. Biol. 37, 1722-1726 (2017).

29. Chang, H. Y. Anatomic demarcation of cells: genes to patterns. Science 326, 12061207 (2009).

30. Pruett, N. D. et al. Changing topographic Hox expression in blood vessels results in regionally distinct vessel wall remodeling. Biol. Open 1, 430-435 (2012).

31. Topouzis, S. \& Majesky, M. W. Smooth muscle lineage diversity in the chick embryo. Two types of aortic smooth muscle cell differ in growth and receptor-mediated transcriptional responses to transforming growth factor-beta. Dev. Biol. 178, 430-445 (1996).

32. Xie, W.-B. B. et al. Smad2 and myocardin-related transcription factor B cooperatively regulate vascular smooth muscle differentiation from neural crest cells. Circ. Res. 113, 76-86 (2013).

33. Madura, J. A. 2nd et al. Regional differences in platelet-derived growth factor production by the canine aorta. J. Vasc. Res. 33, 53-61 (1996).

34. Oh, J., Richardson, J. A. \& Olson, E. N. Requirement of myocardin-related transcription factor-B for remodeling of branchial arch arteries and smooth muscle differentiation. Proc. Natl. Acad. Sci. U. S. A. 102, 15122-15127 (2005).

35. Li, J. et al. Myocardin-related transcription factor B is required in cardiac neural crest for smooth muscle differentiation and cardiovascular development. Proc. Natl. Acad. Sci. U. S. A. 102, 8916-8921 (2005). 
36. Trigueros-Motos, L. et al. Embryological-origin-dependent differences in homeobox expression in adult aorta: role in regional phenotypic variability and regulation of NFkappaB activity. Arterioscler. Thromb. Vasc. Biol. 33, 1248-1256 (2013).

37. Owens, A. P. 3rd et al. Angiotensin II induces a region-specific hyperplasia of the ascending aorta through regulation of inhibitor of differentiation 3. Circ. Res. 106, 611-619 (2010).

38. Sata, M. et al. Hematopoietic stem cells differentiate into vascular cells that participate in the pathogenesis of atherosclerosis. Nat. Med. 8, 403-409 (2002).

39. Caplice, N. M. et al. Smooth muscle cells in human coronary atherosclerosis can originate from cells administered at marrow transplantation. Proc. Natl. Acad. Sci. U. S. A. 100, 4754-4759 (2003).

40. Bentzon, J. F., Sondergaard, C. S., Kassem, M. \& Falk, E. Smooth muscle cells healing atherosclerotic plaque disruptions are of local, not blood, origin in apolipoprotein E knockout mice. Circulation 116, 2053-2061 (2007).

41. Bentzon, J. F. et al. Smooth muscle cells in atherosclerosis originate from the local vessel wall and not circulating progenitor cells in ApoE knockout mice. Arterioscler. Thromb. Vasc. Biol. 26, 2696-2702 (2006).

42. Yu, H. et al. Bone Marrow-Derived Smooth Muscle-Like Cells Are Infrequent in Advanced Primary Atherosclerotic Plaques but Promote Atherosclerosis. Arterioscler. Thromb. Vasc. Biol. 31, 1291-1299 (2011).

43. Dobnikar, L. et al. Disease-relevant transcriptional signatures identified in individual smooth muscle cells from healthy mouse vessels. Nat. Commun. 9, 4567 (2018).

44. Majesky, M. W. Developmental basis of vascular smooth muscle diversity. Arterioscler. Thromb. Vasc. Biol. 27, 1248-1258 (2007).

45. Haimovici, H. The role of arterial tissue susceptibility in atherogenesis. Texas Hear. Inst. J. 18, 81-83 (1991).

46. Benditt, E. P. \& Benditt, J. M. Evidence for a monoclonal origin of human atherosclerotic plaques. Proc. Natl. Acad. Sci. U. S. A. 70, 1753-1756 (1973).

47. Murry, C. E., Gipaya, C. T., Bartosek, T., Benditt, E. P. \& Schwartz, S. M. Monoclonality of smooth muscle cells in human atherosclerosis. Am. J. Pathol. 151, 697-705 (1997).

48. Chung, I. M., Schwartz, S. M. \& Murry, C. E. Clonal architecture of normal and atherosclerotic aorta: implications for atherogenesis and vascular development. Am. J. Pathol. 152, 913-923 (1998).

49. Cheung, C., Bernardo, A. S., Trotter, M. W. B., Pedersen, R. A. \& Sinha, S. Generation of human vascular smooth muscle subtypes provides insight into embryological origin-dependent disease susceptibility. Nat. Biotechnol. 30, 165-173 (2012).

50. Sinha, S. \& Santoro, M. M. New models to study vascular mural cell embryonic origin: implications in vascular diseases. Cardiovasc. Res. 114, 481-491 (2018).

51. Clarke, M. C. H. et al. Chronic apoptosis of vascular smooth muscle cells accelerates atherosclerosis and promotes calcification and medial degeneration. Circ. Res. 102, 1529-1538 (2008).

52. Lee, S. H., Hungerford, J. E., Little, C. D. \& Iruela-Arispe, M. L. Proliferation and differentiation of smooth muscle cell precursors occurs simultaneously during the development of the vessel wall. Dev. Dyn. 209, 342-352 (1997).

53. Poole, J. C., Cromwell, S. B. \& Benditt, E. P. Behavior of smooth muscle cells and formation of extracellular structures in the reaction of arterial walls to injury. Am. J. Pathol. 62, 391-414 (1971).

54. Kocher, O. et al. Phenotypic features of smooth muscle cells during the evolution of 
experimental carotid artery intimal thickening. Biochemical and morphologic studies. Lab. Invest. 65, 459-470 (1991).

55. Chamley-Campbell, J., Campbell, G. R. \& Ross, R. The smooth muscle cell in culture. Physiol. Rev. 59, 1-61 (1979).

56. Kaur, H. et al. Single-cell profiling reveals heterogeneity and functional patterning of GPCR expression in the vascular system. Nat. Commun. 8, 15700 (2017).

57. Pipes, G. C. T., Creemers, E. E. \& Olson, E. N. The myocardin family of transcriptional coactivators: versatile regulators of cell growth, migration, and myogenesis. Genes Dev. 20, 1545-1556 (2006).

58. Rong, J. X., Shapiro, M., Trogan, E. \& Fisher, E. A. Transdifferentiation of mouse aortic smooth muscle cells to a macrophage-like state after cholesterol loading. Proc. Natl. Acad. Sci. U. S. A. 100, 13531-13536 (2003).

59. Vengrenyuk, Y. et al. Cholesterol loading reprograms the microRNA-143/145myocardin axis to convert aortic smooth muscle cells to a dysfunctional macrophagelike phenotype. Arterioscler. Thromb. Vasc. Biol. 35, 535-546 (2015).

60. Allahverdian, S., Chehroudi, A. C., McManus, B. M., Abraham, T. \& Francis, G. A. Contribution of intimal smooth muscle cells to cholesterol accumulation and macrophage-like cells in human atherosclerosis. Circulation 129, 1551-1559 (2014).

61. Andreeva, E. R., Pugach, I. M. \& Orekhov, A. N. Subendothelial smooth muscle cells of human aorta express macrophage antigen in situ and in vitro. Atherosclerosis 135, 19-27 (1997).

62. Wissler, R. W. The arterial medial cell, smooth muscle, or multifunctional mesenchyme? Circulation 36, 1-4 (1967).

63. Alves, R. D. A. M., Eijken, M., van de Peppel, J. \& van Leeuwen, J. P. T. M. Calcifying vascular smooth muscle cells and osteoblasts: independent cell types exhibiting extracellular matrix and biomineralization-related mimicries. BMC Genomics 15, 965 (2014).

64. Durham, A. L., Speer, M. Y., Scatena, M., Giachelli, C. M. \& Shanahan, C. M. Role of smooth muscle cells in vascular calcification: Implications in atherosclerosis and arterial stiffness. Cardiovasc. Res. 114, 590-600 (2018).

65. Sheikh, A. Q., Misra, A., Rosas, I. O., Adams, R. H. \& Greif, D. M. Smooth muscle cell progenitors are primed to muscularize in pulmonary hypertension. Sci. Transl. Med. 7, 308ra159 (2015).

66. Sheikh, A. Q., Saddouk, F. Z., Ntokou, A., Mazurek, R. \& Greif, D. M. Cell Autonomous and Non-cell Autonomous Regulation of SMC Progenitors in Pulmonary Hypertension. Cell Rep. 23, 1152-1165 (2018).

67. Herring, B. P., Hoggatt, A. M., Burlak, C. \& Offermanns, S. Previously differentiated medial vascular smooth muscle cells contribute to neointima formation following vascular injury. Vasc. Cell 6, 21 (2014).

68. Gomez, D. \& Owens, G. K. Reconciling Smooth Muscle Cell Oligoclonality and Proliferative Capacity in Experimental Atherosclerosis. Circ. Res. 119, 1262-1264 (2016).

69. Zhang, L. \& Vijg, J. Somatic Mutagenesis in Mammals and Its Implications for Human Disease and Aging. Annu. Rev. Genet. 52, 397-419 (2018).

70. Jaiswal, S. et al. Clonal Hematopoiesis and Risk of Atherosclerotic Cardiovascular Disease. N. Engl. J. Med. 377, 111-121 (2017).

71. Martin, G. M. \& Sprague, C. A. Clonal senescence and atherosclerosis. Lancet (London, England) 2, 1370-1371 (1972).

72. Munoz-Espin, D. \& Serrano, M. Cellular senescence: from physiology to pathology. Nat. Rev. Mol. Cell Biol. 15, 482-496 (2014). 
73. Campisi, J. Aging, cellular senescence, and cancer. Annu. Rev. Physiol. 75, 685-705 (2013).

74. Kuilman, T., Michaloglou, C., Mooi, W. J. \& Peeper, D. S. The essence of senescence. Genes Dev. 24, 2463-2479 (2010).

75. Grootaert, M. O. et al. Defective autophagy in vascular smooth muscle cells accelerates senescence and promotes neointima formation and atherogenesis. Autophagy 11, 2014-2032 (2015).

76. Matthews, C. et al. Vascular smooth muscle cells undergo telomere-based senescence in human atherosclerosis: effects of telomerase and oxidative stress. Circ. Res. 99, 156-164 (2006).

77. Coppe, J.-P., Desprez, P.-Y., Krtolica, A. \& Campisi, J. The senescence-associated secretory phenotype: the dark side of tumor suppression. Annu. Rev. Pathol. 5, 99-118 (2010).

78. Coppé, J.-P. et al. Senescence-Associated Secretory Phenotypes Reveal CellNonautonomous Functions of Oncogenic RAS and the p53 Tumor Suppressor. PLOS Biol. 6, e301 (2008).

79. Orjalo, A. V, Bhaumik, D., Gengler, B. K., Scott, G. K. \& Campisi, J. Cell surfacebound IL-1alpha is an upstream regulator of the senescence-associated IL-6/IL-8 cytokine network. Proc. Natl. Acad. Sci. U. S. A. 106, 17031-17036 (2009).

80. Gardner, S. E., Humphry, M., Bennett, M. R. \& Clarke, M. C. H. Senescent vascular smooth muscle cells drive inflammation through an interleukin-1 $\alpha$-dependent senescence-associated secretory phenotype. Arterioscler. Thromb. Vasc. Biol. 35, 1963-1974 (2015).

81. Kang, C. et al. The DNA damage response induces inflammation and senescence by inhibiting autophagy of GATA4. Science 349, aaa5612 (2015).

82. Laberge, R.-M. et al. MTOR regulates the pro-tumorigenic senescence-associated secretory phenotype by promoting IL1A translation. Nat. Cell Biol. 17, 1049-1061 (2015).

83. Kang, T.-W. et al. Senescence surveillance of pre-malignant hepatocytes limits liver cancer development. Nature 479, 547-551 (2011).

84. Childs, B. G. et al. Senescent intimal foam cells are deleterious at all stages of atherosclerosis. Science 354, 472-477 (2016).

85. Coppé, J.-P. et al. A Human-Like Senescence-Associated Secretory Phenotype Is Conserved in Mouse Cells Dependent on Physiological Oxygen. PLoS One 5, e9188 (2010).

86. Wang, J. et al. Vascular Smooth Muscle Cell Senescence Promotes Atherosclerosis and Features of Plaque Vulnerability. Circulation 132, 1909-1919 (2015).

87. Shah, A. et al. Defective Base Excision Repair of Oxidative DNA Damage in Vascular Smooth Muscle Cells Promotes Atherosclerosis. Circulation 138, 1446-1462 (2018).

88. Virmani, R., Kolodgie, F. D., Burke, A. P., Farb, A. \& Schwartz, S. M. Lessons From Sudden Coronary Death. Arterioscler. Thromb. Vasc. Biol. 20, 1262-1275 (2000).

89. Yahagi, K. et al. Pathophysiology of native coronary, vein graft, and in-stent atherosclerosis. Nat. Rev. Cardiol. 13, 79-98 (2016).

90. Velican, C. \& Velican, D. Intimal thickening in developing coronary arteries and its relevance to atherosclerotic involvement. Atherosclerosis 23, 345-355 (1976).

91. Ikari, Y., McManus, B. M., Kenyon, J. \& Schwartz, S. M. Neonatal intima formation in the human coronary artery. Arterioscler. Thromb. Vasc. Biol. 19, 2036-2040 (1999).

92. Stary, H. C. et al. A Definition of Initial, Fatty Streak, and Intermediate Lesions of Atherosclerosis. Arter. Thromb. 14, 840-857 (1994).

93. Velican, C. A dissecting view on the role of the fatty streak in the pathogenesis of 
human atherosclerosis: culprit or bystander? Med. Interne 19, 321-337 (1981).

94. Armstrong, M. L., Heistad, D. D., Megan, M. B., Lopez, J. A. \& Harrison, D. G. Reversibility of atherosclerosis. Cardiovasc. Clin. 20, 113-126 (1990).

95. Strong, J. P. et al. Prevalence and extent of atherosclerosis in adolescents and young adults: implications for prevention from the Pathobiological Determinants of Atherosclerosis in Youth Study. JAMA 281, 727-735 (1999).

96. Nakashima, Y., Chen, Y.-X., Kinukawa, N. \& Sueishi, K. Distributions of diffuse intimal thickening in human arteries: preferential expression in atherosclerosis-prone arteries from an early age. Virchows Arch. 441, 279-288 (2002).

97. Nakashima, Y., Wight, T. N. \& Sueishi, K. Early atherosclerosis in humans: Role of diffuse intimal thickening and extracellular matrix proteoglycans. Cardiovasc. Res. 79, 14-23 (2008).

98. Mosse, P. R., Campbell, G. R., Wang, Z. L. \& Campbell, J. H. Smooth muscle phenotypic expression in human carotid arteries. I. Comparison of cells from diffuse intimal thickenings adjacent to atheromatous plaques with those of the media. $L a b$. Invest. 53, 556-562 (1985).

99. Aikawa, M. et al. Human smooth muscle myosin heavy chain isoforms as molecular markers for vascular development and atherosclerosis. Circ. Res. 73, 1000-1012 (1993).

100. Andreeva, E. R., Pugach, I. M. \& Orekhov, A. N. Collagen-synthesizing cells in initial and advanced atherosclerotic lesions of human aorta. Atherosclerosis 130, 133-142 (1997).

101. Skalen, K. et al. Subendothelial retention of atherogenic lipoproteins in early atherosclerosis. Nature 417, 750-754 (2002).

102. Campbell, J. H., Popadynec, L., Nestel, P. J. \& Campbell, G. R. Lipid accumulation in arterial smooth muscle cells. Influence of phenotype. Atherosclerosis 47, 279-295 (1983).

103. Campbell, J. H., Reardon, M. F., Campbell, G. R. \& Nestel, P. J. Metabolism of atherogenic lipoproteins by smooth muscle cells of different phenotype in culture. Arteriosclerosis 5, 318-328 (1985).

104. Kim, D. N., Imai, H., Schmee, J., Lee, K. T. \& Thomas, W. A. Intimal cell massderived atherosclerotic lesions in the abdominal aorta of hyperlipidemic swine. Part 1. Cell of origin, cell divisions and cell losses in first 90 days on diet. Atherosclerosis 56, 169-188 (1985).

105. Ang, A. H., Tachas, G., Campbell, J. H., Bateman, J. F. \& Campbell, G. R. Collagen synthesis by cultured rabbit aortic smooth-muscle cells. Alteration with phenotype. Biochem. J. 265, 461-469 (1990).

106. Lee, R. T. et al. Mechanical strain induces specific changes in the synthesis and organization of proteoglycans by vascular smooth muscle cells. J. Biol. Chem. 276, 13847-13851 (2001).

107. Little, P. J., Tannock, L., Olin, K. L., Chait, A. \& Wight, T. N. Proteoglycans synthesized by arterial smooth muscle cells in the presence of transforming growth factor-beta1 exhibit increased binding to LDLs. Arterioscler. Thromb. Vasc. Biol. 22, 55-60 (2002).

108. Chang, M. Y., Potter-Perigo, S., Tsoi, C., Chait, A. \& Wight, T. N. Oxidized low density lipoproteins regulate synthesis of monkey aortic smooth muscle cell proteoglycans that have enhanced native low density lipoprotein binding properties. $J$. Biol. Chem. 275, 4766-4773 (2000).

109. S.R., L. et al. Extracellular matrix proteomics identifies molecular signature of symptomatic carotid plaques. J. Clin. Invest. 127, 1546-1560 (2017). 
110. Tran-Lundmark, K. et al. Heparan sulfate in perlecan promotes mouse atherosclerosis: roles in lipid permeability, lipid retention, and smooth muscle cell proliferation. Circ. Res. 103, 43-52 (2008).

111. Smith, E. B. \& Slater, R. S. The microdissection of large atherosclerotic plaques to give morphologically and topographically defined fractions for analysis. 1 . The lipids in the isolated fractions. Atherosclerosis 15, 37-56 (1972).

112. Tabas, I., Williams, K. J. \& Borén, J. Subendothelial lipoprotein retention as the initiating process in atherosclerosis: Update and therapeutic implications. Circulation 116, 1832-1844 (2007).

113. Williams, K. J. \& Tabas, I. The response-to-retention hypothesis of early atherogenesis. Arterioscler. Thromb. Vasc. Biol. 15, 551-561 (1995).

114. Thompson, J. C., Tang, T., Wilson, P. G., Yoder, M. H. \& Tannock, L. R. Increased atherosclerosis in mice with increased vascular biglycan content. Atherosclerosis 235, 71-75 (2014).

115. Napoli, C. et al. Fatty streak formation occurs in human fetal aortas and is greatly enhanced by maternal hypercholesterolemia. Intimal accumulation of low density lipoprotein and its oxidation precede monocyte recruitment into early atherosclerotic lesions. J. Clin. Invest. 100, 2680-2690 (1997).

116. Nakagawa, K. \& Nakashima, Y. Pathologic intimal thickening in human atherosclerosis is formed by extracellular accumulation of plasma-derived lipids and dispersion of intimal smooth muscle cells. Atherosclerosis 274, 235-242 (2018).

117. Kockx, M. M. et al. Apoptosis and Related Proteins in Different Stages of Human Atherosclerotic Plaques. Circulation 97, 2307-2315 (1998).

118. Okura, Y. et al. Oxidized low-density lipoprotein is associated with apoptosis of vascular smooth muscle cells in human atherosclerotic plaques. Circulation 102, 2680-2686 (2000).

119. Tulenko, T. N., Chen, M., Mason, P. E. \& Mason, R. P. Physical effects of cholesterol on arterial smooth muscle membranes: evidence of immiscible cholesterol domains and alterations in bilayer width during atherogenesis. J. Lipid Res. 39, 947-956 (1998).

120. Robbins, C. S. et al. Local proliferation dominates lesional macrophage accumulation in atherosclerosis. Nat. Med. 19, 1166-1172 (2013).

121. Ensan, S. et al. Self-renewing resident arterial macrophages arise from embryonic CX3CR1(+) precursors and circulating monocytes immediately after birth. Nat. Immunol. 17, 159-168 (2016).

122. Nahrendorf, M. Myeloid cell contributions to cardiovascular health and disease. Nat. Med. 24, 711-720 (2018).

123. Berliner, J. A. et al. Minimally modified low density lipoprotein stimulates monocyte endothelial interactions. J. Clin. Invest. 85, 1260-1266 (1990).

124. Nelken, N. A., Coughlin, S. R., Gordon, D. \& Wilcox, J. N. Monocyte chemoattractant protein-1 in human atheromatous plaques. J. Clin. Invest. 88, 1121-1127 (1991).

125. Cushing, S. D. et al. Minimally modified low density lipoprotein induces monocyte chemotactic protein 1 in human endothelial cells and smooth muscle cells. Proc. Natl. Acad. Sci. U. S. A. 87, 5134-5138 (1990).

126. Quinn, M. T., Parthasarathy, S., Fong, L. G. \& Steinberg, D. Oxidatively modified low density lipoproteins: a potential role in recruitment and retention of monocyte/macrophages during atherogenesis. Proc. Natl. Acad. Sci. U. S. A. 84, 29952998 (1987).

127. Qiao, J. H. et al. Role of macrophage colony-stimulating factor in atherosclerosis: studies of osteopetrotic mice. Am. J. Pathol. 150, 1687-1699 (1997).

128. Swirski, F. K. et al. Monocyte accumulation in mouse atherogenesis is progressive and 
proportional to extent of disease. Proc. Natl. Acad. Sci. 103, 10340-10345 (2006).

129. Ross, R. et al. Localization of PDGF-B protein in macrophages in all phases of atherogenesis. Science 248, 1009-1012 (1990).

130. Campbell, J. H., Rennick, R. E., Kalevitch, S. G. \& Campbell, G. R. Heparan sulfatedegrading enzymes induce modulation of smooth muscle phenotype. Exp. Cell Res. 200, 156-167 (1992).

131. Ait-Oufella, H. et al. Defective mer receptor tyrosine kinase signaling in bone marrow cells promotes apoptotic cell accumulation and accelerates atherosclerosis. Arterioscler. Thromb. Vasc. Biol. 28, 1429-31 (2008).

132. Ait-Oufella, H. et al. Lactadherin deficiency leads to apoptotic cell accumulation and accelerated atherosclerosis in mice. Circulation 115, 2168-77 (2007).

133. Clarke, M. C. H. H., Talib, S., Figg, N. L. \& Bennett, M. R. Vascular smooth muscle cell apoptosis induces interleukin-1-directed inflammation: Effects of hyperlipidemiamediated inhibition of phagocytosis. Circ. Res. 106, 363-372 (2010).

134. Shaw, P. X. et al. Human-derived anti-oxidized LDL autoantibody blocks uptake of oxidized LDL by macrophages and localizes to atherosclerotic lesions in vivo. Arterioscler. Thromb. Vasc. Biol. 21, 1333-1339 (2001).

135. Schrijvers, D. M., De Meyer, G. R. Y., Kockx, M. M., Herman, A. G. \& Martinet, W. Phagocytosis of apoptotic cells by macrophages is impaired in atherosclerosis. Arterioscler. Thromb. Vasc. Biol. 25, 1256-1261 (2005).

136. Li, S. et al. Defective phagocytosis of apoptotic cells by macrophages in atherosclerotic lesions of ob/ob mice and reversal by a fish oil diet. Circ. Res. 105, 1072-1082 (2009).

137. Tabas, I. Macrophage death and defective inflammation resolution in atherosclerosis. Nat. Rev. Immunol. 10, 36-46 (2010).

138. Naik, V. et al. Sources of cells that contribute to atherosclerotic intimal calcification: An in vivo genetic fate mapping study. Cardiovasc. Res. 94, 545-554 (2012).

139. Sano, H. et al. Functional blockade of platelet-derived growth factor receptor-beta but not of receptor-alpha prevents vascular smooth muscle cell accumulation in fibrous cap lesions in apolipoprotein E-deficient mice. Circulation 103, 2955-2960 (2001).

140. Rekhter, M. D. et al. Type I collagen gene expression in human atherosclerosis. Localization to specific plaque regions. Am. J. Pathol. 143, 1634-1648 (1993).

141. Gomez, D. et al. Interleukin-1 $\beta$ has atheroprotective effects in advanced atherosclerotic lesions of mice. Nat. Med. 24, 1418-1429 (2018).

142. Davies, M. J., Richardson, P. D., Woolf, N., Katz, D. R. \& Mann, J. Risk of thrombosis in human atherosclerotic plaques: role of extracellular lipid, macrophage, and smooth muscle cell content. Br. Heart J. 69, 377-381 (1993).

143. Durgin, B. G. et al. Smooth muscle cell-specific deletion of Col15a1 unexpectedly leads to impaired development of advanced atherosclerotic lesions. Am. J. Physiol. Hear. Circ. Physiol. 312, H943-H958 (2017).

144. Amento, E. P., Ehsani, N., Palmer, H. \& Libby, P. Cytokines and growth factors positively and negatively regulate interstitial collagen gene expression in human vascular smooth muscle cells. Arterioscler. Thromb. a J. Vasc. Biol. 11, 1223-1230 (1991).

145. Rekhter, M. D. Collagen synthesis in atherosclerosis: Too much and not enough. Cardiovasc. Res. 41, 376-384 (1999).

146. Wang, Y. et al. Smooth Muscle Cells Contribute the Majority of Foam Cells in ApoE (Apolipoprotein E)-Deficient Mouse Atherosclerosis. Arterioscler. Thromb. Vasc. Biol. 39 00-00 (2019). doi:10.1161/ATVBAHA.119.312434

147. New, S. E. P. et al. Macrophage-derived matrix vesicles: an alternative novel 
mechanism for microcalcification in atherosclerotic plaques. Circ. Res. 113, 72-77 (2013).

148. Kapustin, A. N. et al. Vascular smooth muscle cell calcification is mediated by regulated exosome secretion. Circ. Res. 116, 1312-1323 (2015).

149. Hutcheson, J. D. et al. Genesis and growth of extracellular-vesicle-derived microcalcification in atherosclerotic plaques. Nat. Mater. 15, 335-343 (2016).

150. Proudfoot, D. et al. Apoptosis regulates human vascular calcification in vitro: evidence for initiation of vascular calcification by apoptotic bodies. Circ. Res. 87, 1055-1062 (2000).

151. Rattazzi, M. et al. Calcification of advanced atherosclerotic lesions in the innominate arteries of ApoE-deficient mice: potential role of chondrocyte-like cells. Arterioscler. Thromb. Vasc. Biol. 25, 1420-1425 (2005).

152. Leroux-Berger, M. et al. Pathologic calcification of adult vascular smooth muscle cells differs on their crest or mesodermal embryonic origin. J. Bone Miner. Res. 26, 15431553 (2011).

153. Espitia, O. et al. Implication of molecular vascular smooth muscle cell heterogeneity among arterial beds in arterial calcification. PLoS One 13, e0191976 (2018).

154. Proudfoot, D., Skepper, J. N., Shanahan, C. M. \& Weissberg, P. L. Calcification of human vascular cells in vitro is correlated with high levels of matrix Gla protein and low levels of osteopontin expression. Arterioscler. Thromb. Vasc. Biol. 18, 379-388 (1998).

155. Steitz, S. A. et al. Smooth muscle cell phenotypic transition associated with calcification: upregulation of Cbfa1 and downregulation of smooth muscle lineage markers. Circ. Res. 89, 1147-1154 (2001).

156. Farrokhi, E., Samani, K. G. \& Chaleshtori, M. H. Oxidized low-density lipoprotein increases bone sialoprotein expression in vascular smooth muscle cells via runt-related transcription factor 2. Am. J. Med. Sci. 349, 240-243 (2015).

157. Al-Aly, Z. et al. Aortic Msx2-Wnt calcification cascade is regulated by TNF-alphadependent signals in diabetic Ldlr-/- mice. Arterioscler. Thromb. Vasc. Biol. 27, 25892596 (2007).

158. Ceneri, N. et al. Rac2 Modulates Atherosclerotic Calcification by Regulating Macrophage Interleukin-1 $\beta$ Production. Arterioscler. Thromb. Vasc. Biol. 37, 328-340 (2017).

159. Zhang, K. et al. Interleukin-18 Enhances Vascular Calcification and Osteogenic Differentiation of Vascular Smooth Muscle Cells Through TRPM7 Activation. Arterioscler. Thromb. Vasc. Biol. 37, 1933-1943 (2017).

160. Cheng, S.-L. et al. Targeted reduction of vascular Msx1 and Msx2 mitigates arteriosclerotic calcification and aortic stiffness in LDLR-deficient mice fed diabetogenic diets. Diabetes 63, 4326-4337 (2014).

161. Hofmann Bowman, M. A. et al. S100A12 in vascular smooth muscle accelerates vascular calcification in apolipoprotein E-null mice by activating an osteogenic gene regulatory program. Arterioscler. Thromb. Vasc. Biol. 31, 337-344 (2011).

162. Nakagawa, Y. et al. Paracrine osteogenic signals via bone morphogenetic protein-2 accelerate the atherosclerotic intimal calcification in vivo. Arterioscler. Thromb. Vasc. Biol. 30, 1908-1915 (2010).

163. Davies, M. J. \& Thomas, A. Thrombosis and acute coronary-artery lesions in sudden cardiac ischemic death. N. Engl. J. Med. 310, 1137-1140 (1984).

164. Pasterkamp, G., Den Ruijter, H. M. \& Libby, P. Temporal shifts in clinical presentation and underlying mechanisms of atherosclerotic disease. Nat. Rev. Cardiol. 14, 21-29 (2016). 
165. Newby, A. C. Dual role of matrix metalloproteinases (matrixins) in intimal thickening and atherosclerotic plaque rupture. Physiol. Rev. 85, 1-31 (2005).

166. Sukhova, G. K. et al. Evidence for increased collagenolysis by interstitial collagenases-1 and -3 in vulnerable human atheromatous plaques. Circulation 99, 2503-2509 (1999).

167. Yu, H. et al. FOXO3a (Forkhead Transcription Factor O Subfamily Member 3a) Links Vascular Smooth Muscle Cell Apoptosis, Matrix Breakdown, Atherosclerosis, and Vascular Remodeling Through a Novel Pathway Involving MMP13 (Matrix Metalloproteinase 13). Arterioscler. Thromb. Vasc. Biol. 38, 555-565 (2018).

168. Falk, E. Plaque rupture with severe pre-existing stenosis precipitating coronary thrombosis. Characteristics of coronary atherosclerotic plaques underlying fatal occlusive thrombi. Br. Heart J. 50, 127-134 (1983).

169. van der Wal, A. C., Becker, A. E., van der Loos, C. M. \& Das, P. K. Site of intimal rupture or erosion of thrombosed coronary atherosclerotic plaques is characterized by an inflammatory process irrespective of the dominant plaque morphology. Circulation 89, 36-44 (1994).

170. Gijsen, F., van der Giessen, A., van der Steen, A. \& Wentzel, J. Shear stress and advanced atherosclerosis in human coronary arteries. J. Biomech. 46, 240-247 (2013).

171. Vengrenyuk, Y. et al. A hypothesis for vulnerable plaque rupture due to stress-induced debonding around cellular microcalcifications in thin fibrous caps. Proc. Natl. Acad. Sci. U. S. A. 103, 14678-14683 (2006).

172. Gordon, D., Reidy, M. A., Benditt, E. P. \& Schwartz, S. M. Cell proliferation in human coronary arteries. Proc. Natl. Acad. Sci. U. S. A. 87, 4600-4604 (1990).

173. O’Brien, E. R. et al. Proliferation in primary and restenotic coronary atherectomy tissue. Implications for antiproliferative therapy. Circ. Res. 73, 223-231 (1993).

174. Han, D. K. et al. Evidence for apoptosis in human atherogenesis and in a rat vascular injury model. Am. J. Pathol. 147, 267-277 (1995).

175. Geng, Y. J. \& Libby, P. Evidence for apoptosis in advanced human atheroma. Colocalization with interleukin-1 beta-converting enzyme. Am. J. Pathol. 147, 251266 (1995).

176. Isner, J. M., Kearney, M., Bortman, S. \& Passeri, J. Apoptosis in human atherosclerosis and restenosis. Circulation 91, 2703-2711 (1995).

177. Bauriedel, G. et al. Role of smooth muscle cell death in advanced coronary primary lesions: implications for plaque instability. Cardiovasc. Res. 41, 480-488 (1999).

178. Clarke, M. C. H. et al. Apoptosis of vascular smooth muscle cells induces features of plaque vulnerability in atherosclerosis. Nat. Med. 12, 1075-1080 (2006).

179. Bennett, M. R., Evan, G. I. \& Schwartz, S. M. Apoptosis of human vascular smooth muscle cells derived from normal vessels and coronary atherosclerotic plaques. J. Clin. Invest. 95, 2266-2274 (1995).

180. Patel, V. A. et al. Defect in insulin-like growth factor-1 survival mechanism in atherosclerotic plaque-derived vascular smooth muscle cells is mediated by reduced surface binding and signaling. Circ. Res. 88, 895-902 (2001).

181. Lyon, C. A., Johnson, J. L., Williams, H., Sala-Newby, G. B. \& George, S. J. Soluble $\mathrm{N}$-cadherin overexpression reduces features of atherosclerotic plaque instability. Arterioscler. Thromb. Vasc. Biol. 29, 195-201 (2009).

182. von der Thusen, J. H. et al. Induction of atherosclerotic plaque rupture in apolipoprotein E-/- mice after adenovirus-mediated transfer of p53. Circulation 105, 2064-2070 (2002).

183. Fernandez-Hernando, C., Jozsef, L., Jenkins, D., Di Lorenzo, A. \& Sessa, W. C. Absence of Akt1 reduces vascular smooth muscle cell migration and survival and 
induces features of plaque vulnerability and cardiac dysfunction during atherosclerosis. Arterioscler. Thromb. Vasc. Biol. 29, 2033-2040 (2009).

184. von der Thüsen, J. H. et al. IGF-1 has plaque-stabilizing effects in atherosclerosis by altering vascular smooth muscle cell phenotype. Am. J. Pathol. 178, 924-934 (2011).

185. Gorenne, I. et al. Vascular smooth muscle cell sirtuin 1 protects against DNA damage and inhibits atherosclerosis. Circulation 127, 386-396 (2013).

186. Tucka, J. et al. Akt1 regulates vascular smooth muscle cell apoptosis through FoxO3a and Apaf1 and protects against arterial remodeling and atherosclerosis. Arterioscler. Thromb. Vasc. Biol. 34, 2421-2428 (2014).

187. Rotllan, N. et al. Genetic Evidence Supports a Major Role for Akt1 in VSMCs During Atherogenesis. Circ. Res. 116, 1744-1752 (2015).

188. Osonoi, Y. et al. Defective autophagy in vascular smooth muscle cells enhances cell death and atherosclerosis. Autophagy 14, 1991-2006 (2018).

189. Zheng, Y., Humphry, M., Maguire, J. J., Bennett, M. R. \& Clarke, M. C. H. Intracellular interleukin-1 receptor 2 binding prevents cleavage and activity of interleukin-1alpha, controlling necrosis-induced sterile inflammation. Immunity 38, 285-295 (2013).

190. Alloza, I. et al. RNAseq based transcriptomics study of SMCs from carotid atherosclerotic plaque: BMP2 and IDs proteins are crucial regulators of plaque stability. Sci. Rep. 7, 1-12 (2017).

191. Gorenne, I., Kavurma, M., Scott, S. \& Bennett, M. Vascular smooth muscle cell senescence in atherosclerosis. Cardiovasc. Res. 72, 9-17 (2006).

192. Liu, Y., Drozdov, I., Shroff, R., Beltran, L. E. \& Shanahan, C. M. Prelamin A accelerates vascular calcification via activation of the DNA damage response and senescence-associated secretory phenotype in vascular smooth muscle cells. Circ. Res. 112, e99-109 (2013).

193. Kolodgie, F. D. et al. Differential accumulation of proteoglycans and hyaluronan in culprit lesions: insights into plaque erosion. Arterioscler. Thromb. Vasc. Biol. 22, 1642-1648 (2002).

194. Franck, G. et al. Flow Perturbation Mediates Neutrophil Recruitment and Potentiates Endothelial Injury via TLR2 in Mice: Implications for Superficial Erosion. Circ. Res. 121, 31-42 (2017).

195. Tricot, O. et al. Relation between endothelial cell apoptosis and blood flow direction in human atherosclerotic plaques. Circulation 101, 2450-2453 (2000).

196. Papakonstantinou, E., Karakiulakis, G., Roth, M. \& Block, L. H. Platelet-derived growth factor stimulates the secretion of hyaluronic acid by proliferating human vascular smooth muscle cells. Proc. Natl. Acad. Sci. U.S.A. 92, 9881-9885 (1995).

197. Ridker, P. M. et al. Antiinflammatory Therapy with Canakinumab for Atherosclerotic Disease. N. Engl. J. Med. 377, 1119-1131 (2017).

198. van der Harst, P. \& Verweij, N. Identification of 64 Novel Genetic Loci Provides an Expanded View on the Genetic Architecture of Coronary Artery Disease. Circ. Res. 122, 433-443 (2018).

199. Miller, C. L. et al. Integrative functional genomics identifies regulatory mechanisms at coronary artery disease loci. Nat. Commun. 7, 12092 (2016).

200. Liu, B. et al. Genetic Regulatory Mechanisms of Smooth Muscle Cells Map to Coronary Artery Disease Risk Loci. Am. J. Hum. Genet. 103, 377-388 (2018).

201. Lo Sardo, V. et al. Unveiling the Role of the Most Impactful Cardiovascular Risk Locus through Haplotype Editing. Cell 175, 1796-1810.e20 (2018).

202. Iyer, D. et al. Coronary artery disease genes SMAD3 and TCF21 promote opposing interactive genetic programs that regulate smooth muscle cell differentiation and 
disease risk. PLoS Genet. 14, e1007681 (2018).

203. Piedrahita, J. A., Zhang, S. H., Hagaman, J. R., Oliver, P. M. \& Maeda, N. Generation of mice carrying a mutant apolipoprotein $\mathrm{E}$ gene inactivated by gene targeting in embryonic stem cells. Proc. Natl. Acad. Sci. U. S. A. 89, 4471-4475 (1992).

204. Ishibashi, S. et al. Hypercholesterolemia in low density lipoprotein receptor knockout mice and its reversal by adenovirus-mediated gene delivery. J. Clin. Invest. 92, 883893 (1993).

205. Schwartz, S. M., deBlois, D. \& O’Brien, E. R. The intima. Soil for atherosclerosis and restenosis. Circ. Res. 77, 445-465 (1995).

206. Campbell, G. R. \& Campbell, J. H. Smooth muscle phenotypic changes in arterial wall homeostasis: implications for the pathogenesis of atherosclerosis. Exp. Mol. Pathol. 42, 139-162 (1985).

207. Ross, R. \& Glomset, J. A. Atherosclerosis and the arterial smooth muscle cell: Proliferation of smooth muscle is a key event in the genesis of the lesions of atherosclerosis. Science 180, 1332-1339 (1973).

208. Ross, R. The pathogenesis of atherosclerosis: a perspective for the 1990s. Nature 362, 801-809 (1993).

209. Schwartz, S. M., Virmani, R. \& Rosenfeld, M. E. The good smooth muscle cells in atherosclerosis. Curr. Atheroscler. Rep. 2, 422-429 (2000).

210. Movat, H. Z., Haust, M. D. \& More, R. H. The morphologic elements in the early lesions of arteriosclerosis. Am. J. Pathol. 35, 93-101 (1959).

211. Buck, R. C. The fine structure of the aortic endothelial lesions in experimental cholesterol atherosclerosis of rabbits. Am. J. Pathol. 34, 897-909 (1958).

212. Wolinsky, H. \& Glagov, S. Structural Basis for the Static Mechanical Properties of the Aortic Media. Circ. Res. 14, 400-413 (1964).

213. Ross, R., Glomset, J. \& Harker, L. Response to injury and atherogenesis. Am. J. Pathol. 86, 675-684 (1977).

214. Davies, M. J. \& Thomas, A. C. Plaque fissuring--the cause of acute myocardial infarction, sudden ischaemic death, and crescendo angina. Heart 53, 363-373 (1985).

215. Etchevers, H. C., Vincent, C., Le Douarin, N. M. \& Couly, G. F. The cephalic neural crest provides pericytes and smooth muscle cells to all blood vessels of the face and forebrain. Development 128, 1059-1068 (2001).

216. Majesky, M. W., Dong, X. R., Regan, J. N. \& Hoglund, V. J. Vascular smooth muscle progenitor cells: Building and repairing blood vessels. Circ. Res. 108, 365-377 (2011).

217. Bentzon, J. F. \& Majesky, M. W. Lineage tracking of origin and fate of smooth muscle cells in atherosclerosis. Cardiovasc. Res. 114, 492-500 (2018).

218. Wasteson, P. et al. Developmental origin of smooth muscle cells in the descending aorta in mice. Development 135, 1823-1832 (2008).

219. Mikawa, T. \& Gourdie, R. G. Pericardial mesoderm generates a population of coronary smooth muscle cells migrating into the heart along with ingrowth of the epicardial organ. Dev. Biol. 174, 221-232 (1996).

220. Hu, Y. et al. Abundant progenitor cells in the adventitia contribute to atherosclerosis of vein grafts in ApoE-deficient mice. J. Clin. Invest. 113, 1258-1265 (2004).

221. Kramann, R. et al. Adventitial MSC-like Cells Are Progenitors of Vascular Smooth Muscle Cells and Drive Vascular Calcification in Chronic Kidney Disease. Cell Stem Cell 19, 628-642 (2016).

222. Zengin, E. et al. Vascular wall resident progenitor cells: a source for postnatal vasculogenesis. Development 133, 1543-1551 (2006).

223. Crisan, M. et al. A Perivascular Origin for Mesenchymal Stem Cells in Multiple Human Organs. Cell Stem Cell 3, 301-313 (2008). 
224. Tigges, U., Komatsu, M. \& Stallcup, W. B. Adventitial pericyte progenitor/mesenchymal stem cells participate in the restenotic response to arterial injury. J. Vasc. Res. 50, 134-144 (2013).

225. Parmacek, M. S. Myocardin: dominant driver of the smooth muscle cell contractile phenotype. Arterioscler. Thromb. Vasc. Biol. 28, 1416-1417 (2008).

226. Yoshida, T., Yamashita, M., Horimai, C. \& Hayashi, M. Smooth muscle-selective inhibition of nuclear factor- $\kappa \mathrm{B}$ attenuates smooth muscle phenotypic switching and neointima formation following vascular injury. J. Am. Heart Assoc. 2, e000230e000230 (2013).

227. Zhou, A.-X. et al. C/EBP-Homologous Protein (CHOP) in Vascular Smooth Muscle Cells Regulates Their Proliferation in Aortic Explants and Atherosclerotic Lesions. Circ. Res. 116, 1736-1743 (2015).

228. Cordes, K. R. et al. miR-145 and miR-143 regulate smooth muscle cell fate and plasticity. Nature 460, 705-710 (2009).

229. Raines, E. W. The extracellular matrix can regulate vascular cell migration, proliferation, and survival: relationships to vascular disease. Int. J. Exp. Pathol. 81, 173-182 (2000).

230. Opitz, F., Schenke-Layland, K., Cohnert, T. U. \& Stock, U. A. Phenotypical plasticity of vascular smooth muscle cells-effect of in vitro and in vivo shear stress for tissue engineering of blood vessels. Tissue Eng. 13, 2505-2514 (2007).

231. Liu, R. et al. Ten-eleven translocation-2 (TET2) is a master regulator of smooth muscle cell plasticity. Circulation 128, 2047-2057 (2013).

232. Chen, J. et al. Histone demethylase KDM3a, a novel regulator of vascular smooth muscle cells, controls vascular neointimal hyperplasia in diabetic rats. Atherosclerosis 257, 152-163 (2017).

233. Zhuang, J. et al. The yin-yang dynamics of DNA methylation is the key regulator for smooth muscle cell phenotype switch and vascular remodeling. Arterioscler. Thromb. Vasc. Biol. 37, 84-97 (2017).

234. Greissel, A. et al. Alternation of histone and DNA methylation in human atherosclerotic carotid plaques. Thromb. Haemost. 114, 390-402 (2015).

235. Lino Cardenas, C. L. et al. An HDAC9-MALAT1-BRG1 complex mediates smooth muscle dysfunction in thoracic aortic aneurysm. Nat. Commun. 9, 1009 (2018).

236. Fiedler, J. et al. Non-coding RNAs in vascular disease - from basic science to clinical applications: scientific update from the Working Group of Myocardial Function of the European Society of Cardiology. Cardiovasc. Res. 114, 1281-1286 (2018).

237. Das, S. et al. A Novel Angiotensin II-Induced Long Noncoding RNA Giver Regulates Oxidative Stress, Inflammation, and Proliferation in Vascular Smooth Muscle Cells. Circ. Res. 123, 1298-1312 (2018).

238. Ballantyne, M. D. et al. Smooth Muscle Enriched Long Noncoding RNA (SMILR) Regulates Cell Proliferation. Circulation 133, 2050-2065 (2016).

239. Nurnberg, S. T. et al. Coronary Artery Disease Associated Transcription Factor TCF21 Regulates Smooth Muscle Precursor Cells That Contribute to the Fibrous Cap. PLoS Genet. 11, e1005155 (2015).

240. Evrard, S. M. et al. Endothelial to mesenchymal transition is common in atherosclerotic lesions and is associated with plaque instability. Nat. Commun. 7, 11853 (2016). 


\title{
Figure legends
}

\author{
Figure 1: Overview of the role of vascular smooth muscle cells (VSMCs) in \\ atherosclerosis \\ VSMCs are a major source of plaque cells and extra-cellular matrix (ECM) at all stages of \\ atherosclerosis and contribute to numerous processes throughout the disease.
}

\section{Figure 2: VSMCs in early atherosclerosis}

Summary of the role of VSMCs in early atherosclerosis (progression from diffuse intimal thickening to pathological intimal thickening). VSMCs are the predominant cell type and source of atherogenic, lipid (particularly LDL)-retentive extra-cellular matrix in early atherosclerosis. Retained LDL is susceptible to modifications, such as oxidation (to OxLDL). Uptake of OxLDL by VSMCs leads to foam cell formation and death by apoptosis. Activated VSMC secrete chemokines and contribute to recruitment of monocytes, which differentiate to macrophages. Progression to PITs is typically associated with decreased VSMC marker positive cell content (such as smooth muscle alpha actin positive cells, $\alpha \mathrm{SMA}+$ ) and increased macrophage marker positive cells (such as CD68+ cells), likely reflecting a combination of VSMC death and VSMC phenotype switching to macrophage like cells (as a consequence of decreased MYOCD and increased KLF4).

\begin{abstract}
Abbreviations: ABCA1, ATP-binding cassette transporter 1 ApoB, apolipoprotein B; CCL2, CC motif chemokine 2 (also known as MCP-1); CCL5, CC motif chemokine 5 (also known as RANTES); CXCL1, CXC motif chemokine 1 (also known as GRO $\alpha$ ); DIT, diffuse intimal thickening, ECM, extra-cellular matrix; ECs, endothelial cells; ICAM1, intercellular adhesion molecule 1; KLF4, Krüppel like factor 4; LDL, low density lipoprotein; MYOCD, myocardin; PIT, pathological intimal thickening; SR, scavenger receptor; VCAM1, vascular cell adhesion molecule 1; VSMCs, vascular smooth muscle cells.
\end{abstract}

\section{Figure 3: VSMCs in late atherosclerosis}

Summary of the role of VSMCs in late atherosclerosis (progression from pathological intimal thickening to fibroatheroma). This phase of atherosclerosis is characterised by the elaboration of the fibrous cap by VSMCs, and the necrotic core, which is the consequence of defective efferocytosis of apoptotic cells (mostly VSMCs and macrophages). Through phenotype switching, VSMCs contribute to many different plaque cell phenotypes, including the extra-cellular matrix -producing cells of the fibrous cap, macrophage-like cells, foam cells, mesenchymal stem cell-like and osteochondrogenic cells. VSMC also contribute to calcification through a number of mechanisms, including apoptosis and osteochondrogenic conversion.

\begin{abstract}
Abbreviations: $\alpha$ SMA, smooth muscle alpha actin; DAMPs, damage associated molecular patterns; ECM, extracellular matrix; IL-1 $\alpha$, interleukin-1 alpha; KLF4, Krüppel like factor 4; LDL, low density lipoprotein; MSClike, mesenchymal stem cell-like; MYOCD, myocardin; PDGF, platelet derived growth factor; PIT, pathological intimal thickening; VSMCs, vascular smooth muscle cells.
\end{abstract}

\section{Figure 4: VSMCs in clinical sequalae of atherosclerosis}

Summary of the role of VSMCs in plaque rupture and plaque erosion, the two major processes underlying thrombosis and hence the clinical sequalae of atherosclerosis. 
Abbreviations: ECM, extra-cellular matrix; EC, endothelial cell; IL-, interleukin-; MMPs, matrix metalloproteinases; SASP, senescence associated secretory phenotype; TLR, Toll like receptor; VSMCs, vascular smooth muscle cells.

Table 1: Lineage tracing studies in atherosclerosis

\section{Glossary}

Clonal expansion - proliferation of a single or limited number of ancestral cells

Foam cell - lipid laden cells with a foamy appearance

Lineage tracing - technique of following the fate of labelled cells to enable identification of progeny cells

Mesenchymal stem cells - multipotent stromal cells

Osteochondrogenic cells - cells capable of generating osteocytes and or chondrocytes

Phenotype switching - process by which VSMCs alter phenotype, often infered through decreased expression of VSMC-specific contractile genes and or increased expression of markers typical of synthetic VSMCs or other cell-types

Response to retention hypothesis - hypothesis that sub-endothelial retention of lipid, in the form of lipoproteins, is the initial step in atherogenesis

Shelterin complex - multi-protein complex (including TRF2) which binds the repetitive sequences of telomeric DNA, protecting against DNA damage

Vulnerable plaque - plaque with a phenotype associated with increased risk of rupture, also known as thin-cap fibroatheromas, defined by a thin fibrous cap (of less than $65 \mu \mathrm{m}$ ) and large necrotic core

\section{Abbreviations}

AngII

ApoB

aSMA

CAD

CArG box

CyToF

DIT

DDR

DT

ECM

GAG

GWAS

MSC

MMPs

oxLDL

ROS

SASP

$\mathrm{Sa} \beta \mathrm{G}$

Shelterin

X-Gal

48

49

Author contributions 
G.L.B., H.F.J. and M.C.H.C. wrote the manuscript. H.F.J. and M.C.H.C. contributed equally.

2 All the authors researched data for the article, discussed its content, reviewed the manuscript 3 for important intellectual content, and edited the manuscript before submission.

4

5

6

7

8

9

10

11

12

13

14

15

16

17

18

19

20

21

22

23

24

25

26

27

28

\section{Competing interests}

The authors declare no competing interests.

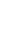

(1)

